\title{
Index to Volume 119
}

\section{Compiled by Leslie Cody}

Abies amabilis, 160

balsamea, 129,255,381,457,570

lasiocarpa, 186

Acanthomyops subglaber, 39

Accipiter cooperii, 379

striatus, 377

Acer negundo, 220,438

Acer pensylvanicum, 129

Acer rubrum, 17,240,386

Acer saccharinum, 129

Acer saccharum, 38,129,332,378,386,457

Acer spicatum, 457

Achaearanea ohlerti, 269

tabulata, 269

tepidariorum, 269

Achigan à grande bouche, 363

à petite bouche, 363

Achillea millefolium, 248

millefolium $\mathrm{f}$. rosea, 438

ptarmica, 438

Acjaearamea tabulata, 272

Aconitum sp., 438

Actaea rubra f. neglecta, 438

Actitis macularia, 69,379

Aculepeira carbonarioides, 256

Adkins-Giese, C.L., and F.J. Cuthbert. Woodpecker Nest Tree Characteristics in Upper Midwestern Oak Forests, 367

Aegithalus caudata, 120

Aegolius acadicus, 367,578 funereus, 367,578

Aegolius acadicus, Caching Behavior by Wintering Northern Saw-Whet Owls, 578

Aesculus hippocastanum, 344

Agelaius phoeniceus, 69

Agelenopsis, 95

utahana, 256

Agroeca ornata, 266

Agropyron sericeum, 279

smithii, 220

trachycaulum, 78, 279

Agrostis humilis, 417

scabra, 394

thurberiana, 420

Agyneta sp., 259

allosubtilis, 259

amersaxatilis, 259

dynica, 259

fabra, 259

jacksoni, 259

olivacea, 259

simplex, 259

Aix sponsa, 69,379,548

Alaska, Attempted Predation of a Child by a Gray Wolf, Canis lupus, near Icy Bay, 197
Alaska Beaufort Sea Lagoons, 1999-2002, Long-tailed Duck, Clangula hyemalis, Eider, Somateria spp., and Scoter, Melanitta spp., Distributions in Central, 181

Alberta Alfalfa Fields, Body Weights of Adult and Juvenile Northern Pocket Gophers, Thomomys talpoides, in Central, 551

Alberta's Tardigrade Fauna, Water-bears from the Rocky Mountains: A First Look at, 586

Alberta, Travel Rates of Wolves, Canis lupus, in Relation to Ungulate Kill Sites in Westcentral, 573

Alces alces, 43,50,136,168,186,194,197,323,330,381,574

Alces alces, and Beaver, Castor canadensis, in Algonquin Park, Ontario, 1860-2004, Reconstructing Changes in Abundance of White-tailed Deer, Odocoileus virginianus, Moose, 330

Alces alces, in Central Interior British Columbia, Winter Habitat Use by Moose, 186

Alder, 96,591

Speckled, 65

Alewife, 18

Alfalfa, 175,551

Alfalfa Fields, Body Weights of Adult and Juvenile Northern Pocket Gophers, Thomomys talpoides, in Central Alberta, 551

Alfalfa Fields Inhabited by Northern Pocket Gophers, Thomomys talpoides, Long-tailed Weasel, Mustela frenata, Movements and Diggings in, 175

Alga, Marine Green, 82

Alga Percursaria percursa from Hypersaline Springs in the Middle of the North American Continent, Identification of a Marine Green, 82

Alisma plantago-aquatica var. americanum, 279 triviale, 277

Allan, M., 453

Allomengea dentisetis, 259

Alnus spp., 96,386,591

incana, 389

incana americana, 96

incana ssp. rugosa, 65,129,388

rugosa, 96

Alopecosa aculeata, 266

Alopecurus arundinaceus, 438

geniculatus, 418

occidentalis, 438

pratensis, 418

Alopex lagopus, 120,323

Alosa pseudoharengus, 21

Amara aulica, 271

Amaranthus spp., 548

Amaurobius borealis, 256

Ambloplites rupestris, 21

Ambylpone pallipes, 39

Ambystoma gracile, 292 macrodactylum, 291 
Ambystoma macrodactylum, in the Oregon Cascade Range, Apparent Predation by Gray Jays, Perisoreus canadensis, on Long-toed Salamanders, 291

Ameiurus nebulosus, 21,295,360

Amelanchier alnifolia, 78

laevis, 240

lucida, 239

Amia calva, 21

Ammodramus leconteii, 69

Ammodytes americanus, 216

Ammophila harti, 9

Amsinckia menziesii, 438

Anas acuta, 69,548

americana, 69

clypeata, 69,548

crecca, 69,548

discors, 69,379,548

platyrhynchos, 69,379,541,546

rubripes, 69

strepera, 548

Anas platyrhynchos, Use of Radio-Telemetry to Test for Investigator Effects on Nesting Mallards, 541

Anax junius, 234

Andersen, D.E., 208

Andrena spp., 53,249

Andrenid, 53

Andromeda polifolia, 210

Anemone parviflora, 276 richardsonii, 276

Anemone, Plumose, 355

Angelica lucida, 417

Angelica, Seacoast, 433

Anguilla rostrata, 294,351

Ant Species (Hymenoptera: Formicidae) Richness at the Mont St. Hilaire Biosphere Reserve, Québec, The Effect of Human Activity on, 38

Antennaria isolepis, 276 pulcherrima, 417

Anthus spragueii, 490

Antistea brunnea, 259

Apamea amputatrix, 12 ophiogramma, 12

Aphaenogaster rudis, 39

Aphileta misera, 259

Aphragmus eschscholtzianus, 417

Apis mellifera, 53,249

Aplodinotus grunniens, 21

Apocynum androsaemifolium, 417

Arabidopsis salsuginea, 417

Arabis boivinii, 417

Aralia nudicaulis, 44

Araneus corticarius, 256

diadematus, 255

displicatus, 257

groenlandicola, 257

marmoreus, 257

nordmanni, 257

saevus, 257

solitarius, 257

trifolium, 257

washingtoni, 257

Araniella displicata, 257

proxima, 257

Arcella, 403

arenaria var. grandis, 410 artocrea, 410

artocrea ssp. pseudocatinus, 410

bathystoma, 410

crenata, 410

formosa, 403

leidyana, 410

megastoma, 410

rota, 410

Arcella formosa n. sp.: Two New Species of Testate Rhizopods (Arcellinida, Protozoa) from Remnant Wetlands in Ontario, Canada, Cyclopyxis acmodonta n. sp. and, 403

Archilochus colubris, 70,379

Arctosa alpigena, 266

emertoni, 267

insignita, 267

lama, 267

quinaria, 267

raptor, 267

rubicunda, 267

virgo, 267

Arctostaphylos spp., 210

alpina, 277

uva-ursi, 240

Ardea herodias, 69,379

Argenna obesa, 258

Argyneta cauta, 259 decora, 259

Arnica alpina ssp. attenuata, 289

angustifolia ssp. attenuata, 277

chamissonis, 277

diversifolia, 417

latifolia, 417

Arnica, Diverse, 434

Mountain, 435

Aronia melanocarpa, 240

Arrow-grass, Seaside, 279

Artemisia sp., 220,532

absinthium, 438

dracunculus, 438

furcata, 277

hyperborea, 289

tilesii, 277,417

Asclepias syriaca, 525

Ash, Mountain, 570

Asio flammeus, 70,400 otus, 395

Asio otus: A Review of North American Banding, Long-eared Owls, 395

Aspen, 175,369,401

Big-toothed, 371

Quaking, 370

Trembling, 65,135,186,220,370,381,442,451,574

Asphodel, Sticky False, 428

Aspicilia, 78 caesiocinerea, 78

Aster brachyactis, 418

curtus, 245

hesperius, 439

lanceolatus, 390

lanceolatus $\mathrm{ssp}$. hesperius, 439

puniceus, 276,388

pygmaeus, 277

sibiricus, 277

yukonensis, 418 
Aster, Lindley's American, 435

Purple-stemmed, 289

Rayless, 435

Western Willow, 439

White-top, 248

Yukon, 435

Yukon-American, 435

aster du Saint-Laurent, 556

Astragalus alpinus, 417,506

australis, 277

eucosmus, 276

eucosmus f. albinus, 276

richardsonii, 287

Athene culicularia, 490

Athyrium filix-femina var. cyclosorum, 417

Atkinson, J., Review by, 305

Attix, L., 455

Auditor's Report, 478

Aulnes rugueux, 129

Avena sativa, 418

Aythya collaris, 69 valisineria, 546

Babin, T., 453

Badger, 584

North American, 442

Badiou, P.H., 82

Baeolophus bicolor, 374

Baggs, E.M., 323

Bakeapple, 412

Balaenoptera acutorostrata, 214 musculus, 214 physalus, 214

Balaenoptera acutorostrata, in the Saguenay-St. Lawrence National Marine Park, Novel Surface Feeding Tactics of Minke Whales, 214

Ballard, W.B., 584

Baneberry, Red, 438

Barbotte brune, 360

Barker, M.E., 58

Barnes, D.M. Possible Tool Use by Beavers, Castor canadensis, in a Northern Ontario Watershed, 441

Bartramia longicauda, 69

Baryphyma kulczynskii, 259

Bass, Largemouth, 21

Rock, 21

Smallmouth, 21,295,353

White, 21

Bassaniana utahensis, 270

Basswood, 369

Bat, Big Brown, 132

Hoary, 132

Little Brown, 444,589

Bat by American Crows, Corvus brachyrhynchos, Predation of a, 443

Bat, Lasiurus cinereus, Use of a Bridge for Day Roosting by the Hoary, 132

Bathyphantes brevipes, 259

brevis, 259

canadensis, 260

concolor, 260

crosbyi, 260

gracilis, 260

pallidus, 260

pullatus, 263 reprobus, 260

rufulus, 263

simillimus, 260

sp. reprobus, 260

Bats, Myotis lucifugus, in a Rodent Trap in the Boreal Forest, Mortality of Little Brown, 589

Batt, J.H., 355

Beak-rush, White, 281

Bear, Black, 50,164,298,323,339,381,574

Brown, 339,449

Grizzly, 574

Bear, Ursus americanus, Ecology on the Northeast Coast of Labrador, Black, 164

Bears, Ursus arctos, from Coastal British Columbia, Observations of Autumn Courtship and Breeding in Brown, 449

Bearberry, 210 Alpine, 288

Beaudoin, A.B., Reviews by, 150,465,596,603

Beaver, 176,197,330,385,441,591

Beaver, Castor canadensis, Flooding on a Small Shore Fen in Southwestern Quebec, Consequences of, 385

Beaver, Castor canadensis, in Algonquin Park, Ontario, 18602004, Reconstructing Changes in Abundance of Whitetailed Deer, Odocoileus virginianus, Moose, Alces alces, and, 330

Beavers, Castor canadensis, Feeding on Salmon Carcasses: Opportunistic Use of a Seasonally Superabundant Food Source, 591

Beavers, Castor canadensis, in a Northern Ontario Watershed, Possible Tool Use by, 441

Bedstraw, Sweet-scented, 434

Beech, American, 38,332,378

Beetle, 271

Leaf, 88

Mountain Pine, 186

Beetles), in North America (Coleoptera: Chrysomelidae, Chrysomelinae), New Distribution Records and Biogeography of Calligrapha Species (Leaf, 88

Bembix americana, 10 pallidipicta, 9

Bennett, B.A., 417

Bennett, K.E., E.M. Baggs, J.R. Finney-Crawley, and M. McGrath. Analysis of the Parasites of a Mid-winter Population of the Snowshoe Hare, Lepus americanus, on Insular Newfoundland During a Cyclical Peak, 323

Bennett, R., Review by, 303

Bentgrass, Alpine, 420

Berry, C.R. Jr., 219

Berry, Thorny Buffalo, 220

Betula sp., 96,186,255,386,413,591

alleghaniensis, 332,386,457,570

cordifolia, 570

glandulosa, 210

lenta, 96

papyrifera, 41,96,103,129,381,386,451,457,569

populifolia, 129,240

Bidens sp., 388,548

сегnua, 394

connata, 390

Bidensomela, 89

Bilberry, Alpine, 412

Bind-weed, 428

Birch, 186,255,591 
Dwarf, 210,413

Heart-leaved, 570

Paper, 41,103,371,569

White, 381,386,451

Yellow, 332,386,570

Bistort, 283

Bitter-cress, 248

Bittern, American, 69

Blackbird, Brewer's, 69

Red-winged, 69

Bladderwort, Greater, 137

Lesser, 434

Blarina brevicauda, 456,578

Blarina brevicauda, Apparently Killed by Red Squirrel, Tamiasciurus hudsonicus, Short-tailed Shrew, 456

Blueberries, Vaccinium angustifolium Ait. and V. myrtilloides Michx. (Ericacaeae), in the Boreal Forest, Pollination and Breeding System of Lowbush, 48

Blueberry, 44 Lowbush, 48

Bluegill, 20

Bluet, 234

Familiar, 234

Blysmopsis rufa, 417

Blysmus rufus, 422

Boal, C.W. and D.E. Andersen. Microhabitat Characteristics of Lapland Longspur, Calcarius lapponicus, Nests at Cape Churchill, Manitoba, 208

Bobolink, 70

Boeckner, M.J. and H.C. Proctor. Water-bears from the Rocky Mountains: A First Look at Alberta's Tardigrade Fauna, 586

Bogbean, 433

Bombus spp., 48

bifarius, 249

flavifrons, 249

ternarius, 56

terricola, 56

Bombycilla cedrorum, 70

Bombylius major, 249

Bonasa umbellus, 44,71,379

Bonasia bonasia, 120

Bondrup-Nielsen, S., 164

Boreal Dip Net/L'Epuisette Boreale: Newsletter of the Canadian Amphibian and Reptile Conservation Network/ Reseau Canadien de Conservation des Amphibiens et des Reptiles, The, 319,473

Botaurus lentiginosus, 69

Bouleau blanc, 129,457 gris, 129

jaune, 457

Bouteloua gracilis, 220

Bowfin, 18

Boyd, D.K., 446

Boyd, H., 483

Boyle, H.F., 6

Brachinus sp., 16

Brachymyrmex depilis, 38

Bradford, R.G., 294

Brant, Eastern High Arctic, 486

Branta bernicla hrota, 486 canadensis, 69,379,483 canadensis canadensis, 483 canadensis interior, 483 hutchinsii, 483
Branta canadensis, and Cackling Geese, Branta hutchinsii, in the Eastern Canadian Arctic, Breeding and NonBreeding Range of Canada, 483

Branta hutchinsii, in the Eastern Canadian Arctic, Breeding and Non-Breeding Range of Canada, Branta canadensis, and Cackling Geese, 483

Brasenia schreberi, 137

Braya glabella, 277 humilis, 277

British Columbia, Body Size Distribution and Frequency of Anthropogenic Injuries of Bluntnose Sixgill Sharks, Hexanchus griseus, at Flora Islets, 537

British Columbia, Insect Visitation to Wildflowers in the Endangered Garry Oak, Quercus garryana, Ecosystem of, 245

British Columbia, Observations of Autumn Courtship and Breeding in Brown Bears, Ursus arctos, from Coastal, 449

British Columbia's Coastal Archipelago, Facts from Faeces: Prey Remains in Wolf, Canis lupus, Faeces Revise Occurrence Records for Mammals of, 192

British Columbia, The Distribution and Habitat Selection of Introduced Eastern Grey Squirrels, Sciurus carolinensis, in, 343

British Columbia, Winter Habitat Use by Moose, Alces alces, in Central Interior, 186

Brodiaea, Howell's, 248

Brome, Japanese, 421

Bromus japonicus, 417

Brooklime, American, 434

Broom, Scotch, 245

Broomrape, Clustered, 434

Bubo scandiacus, 211 virginianus, $62,71,395,578$

Bucephala clangula, 69

Buchanan, L., 453

Buckbean, 433

Buckbrush, 220

Buckwheat, Wild, 428

Buddle, C.M., 38

Buffalo, Bigmouth, 18

Bufo boreas, 291

Bullfinch, 120

Bullhead, Brown, 21,295

Bulrush, 16

Common Three-Square, 439

Great, 17

Hard-stemmed, 427

Red, 422

Bumblebee, 48

Bunchberry, 44,412

Bunting, Snow, 120

Bur-reed, 16,137

Bury, R.B., 291

Buteo lineatus, 377 platypterus, 71,377 regalis, 490

Butter-and-eggs, 14

Buttercup, Western, 248

White Water, 285

Butterfly, Monarch, 525

Cactus, Prickly Pear, 220

Calamagrostis canadensis, 77 lapponica, 277 
neglecta, 279

purpurascens, 277

stricta ssp. stricta, 277

Calcarius lapponicus, 208

Calcarius lapponicus, Nests at Cape Churchill, Manitoba, Microhabitat Characteristics of Lapland Longspur, 208

Calidris alpina, 379

minutilla, 379

Calla palustris, 388

Calliergon stramineum, 388

Calligrapha, 88

alni, 89

alnicola, 89

amator, 89

amelia, 89

androwi, 95

apicalis, 89

bidenticola, 91

californica, 92

cephalanti, 95

confluens, 92

dislocata, 95

dolosa, 96

floridana, 95

fulvipes, 95

ignota, 89

incisa, 96

knabi, 92

lunata, 96

multiguttata, 95

multipunctata, 96

ostryae, 92

philadelphica, 93

pnirsa, 96

praecelsis, 96

pruni, 96

rhoda, 93

rowena, 89

scalaris, 93

sensu stricta, 89

serpentina, 95

sigmoidea, 96

spiraea, 94

stolonifera, 96

suturella, 94

sylvia, 95

tiliae, 95

verrucosa, 95

vicina, 95

virginea, 96

wickhami, 95

Calligrapha Species (Leaf Beetles), in North America (Coleoptera: Chrysomelidae, Chrysomelinae), New

Distribution Records and Biogeography of, 88

Callioplus euoplus, 256 tibialis, 256

Callitriche hermaphroditica, 277

Callobius bennetti, 256

Calophasia lunula, 12

Caltha natans, 276

palustris var. arctica, 277

Camas, Common, 245

Camassia quamash, 245

Camomile, Sea-shore, 290
Campanula rapunculoides, 438

rotundifolia, 417

uniflora, 276

Camponotus nearcticus, 39

novaeboracensis, 39

pennsylvanicus, 39

Campostoma anomalum, 21

Campylium sp., 389 polygamum, 388

Canada? A Specimen from the Arctic Archipelago, Northwest Territories Links Two Allopatric Species of Alkali Grass, Puccinellia, Addition to the Flora of, 497

Canada, Branta canadensis, and Cackling Geese, Branta hutchinsii, in the Eastern Canadian Arctic, Breeding and Non-Breeding Range of, 483

Canada, Cyclopyxis acmodonta $\mathrm{n}$. sp. and Arcella formosa $\mathrm{n}$. sp.: Two New Species of Testate Rhizopods (Arcellinida, Protozoa) from Remnant Wetlands in Ontario, 403

Canada, First Record of the Plains Minnow, Hybognathus placitus, in, 219

Canada, Papillate Watermeal, Wolffia brasiliensis, in Eastern Ontario: An Addition to the Flora of, 137

Canada III, Additions and Range Extensions to the Vascular Plant Flora of the Continental Northwest Territories and Nunavut, 276

Canadian Arctic, Breeding and Non-Breeding Range of Canada, Branta canadensis, and Cackling Geese, Branta hutchinsii, in the Eastern, 483

Canadian Field-Naturalist, Advice for Contributors to The, $158,482,642$

Canadian Field-Naturalist 119(1), Erratum: The, 320

Canadian Journal: Wildlife Afield, New, 156

Canadian Species at Risk, 613

Canadian Waters, First Records of Long-beaked Common Dolphins, Delphinus capensis, in, 110

Canis latrans, 1,134,139,161,200,324,381,574,580,584 lupus, $1,101,135,192,197,381,442,446,573$

lupus beothucus, 323 lycaon, 139,330

Canis latrans, in Labrador, Evidence of Range Expansion of Eastern Coyotes, 381

Canis latrans, in New York, Chronology of Range Expansion of the Coyote, 1

Canis latrans, Record Size Female Coyote, 139

Canus lupus, Den and Associated Human Activity in the Southwestern Yukon Territory, An Ancient Wolf, 135

Canis lupus, Faeces Revise Occurrence Records for Mammals of British Columbia's Coastal Archipelago, Facts from Faeces: Prey Remains in Wolf, 192

Canis lupus, in Relation to Ungulate Kill Sites in Westcentral Alberta, Travel Rates of Wolves, 573

Canis lupus, in the Wild, Longevity and Productivity of Three Wolves, 446

Canis lupus, near Icy Bay, Alaska, Attempted Predation of a Child by a Gray Wolf, 197

Canvasback, 546

Capelin, 215,355

Capercaillie, 120

Cappuccino, N., 525

Carassius auratus, 21

Carbyn, S., 237

Cardamine sp., 248

bellidifolia, 276

pratensis, 276 
Cardinal, Northern, 71

Cardinalis cardinalis, 71

Carduelis flammea, 120

hornemanni, 120

pinus, 71

tristis, 71

Carex sp., 16,186,388,548,591

aquatilis, 210

atherodes, 77

bebbii, 277,394,417

brunnescens, 417

buxbaumii, 417

canescens, 276,417

comosa, 137

cryptolepis, 388

eburnea, 417

enanderi, 425

lasiocarpa, 389,417

laxa, 417

lenticularis var. dolia, 417

lenticularis var. lipocarpa, 417

livida, 417

media, 276

microglochin, 417

microptera, 417

nardina, 277,418

nigricans, 417

parryana, 418

phaeocephala, 418

pseudo-cyperus, 389

rariflora, 276

rotundata, 276

sartwellii, 417

scirpoidea, 276

siccata, 417

subspathacea, 277

supina ssp. spaniocarpa, 277

tonsa var. rugosperma, 240

vaginata, 277

Caribou, 43,119,168,201,355,381

Peary, 120

Woodland, 135,573

Carorita limnaea, 260

Carp, Common, 18,546

Carpiodes cyprinus, 21

Carpodacus purpureus, 70

Carya spp., 343

cordiformis, 369

ovata, 369

Cassiope stellariana, 433

tetragona, 506

Castanea dentata, 343

Castilleja miniata, 418 parviflora, 418 unalaschcensis, 418

Castor canadensis, 176,197,330,385,441,591

Castor canadensis, Feeding on Salmon Carcasses: Opportunistic Use of a Seasonally Superabundant Food Source, Beavers, 591

Castor canadensis, Flooding on a Small Shore Fen in Southwestern Quebec, Consequences of Beaver, 385

Castor canadensis, in Algonquin Park, Ontario, 1860-2004, Reconstructing Changes in Abundance of White-tailed Deer, Odocoileus virginianus, Moose, Alces alces, and Beaver, 330
Castor canadensis, in a Northern Ontario Watershed, Possible Tool Use by Beavers, 441

Caswell, P., 417

Catbird, Gray, 70

Catchfly, Balkan, 439

Catfish, Channel, 21

Catharus fuscescens, 70 guttatus, 70 ustulatus, 70

Catling, P.M. Additions to the Flora of the Continental Northwest Territories from the Great Slave Lake Area, 437

Catling, P.M. A Potential for the Use of Dragonfly (Odonata) Diversity as a Bioindicator of the Efficiency of Sewage Lagoons, 233

Catling, P.M. Identification and Status of the Introduced Black Pine, Pinus nigra, and Mugo Pine, Pinus mugo, in Ontario, 224

Catling, P.M., Review by, 597

Catling, P.M. and S. Carbyn. Invasive Scots Pine, Pinus sylvestris, Replacing Corema, Corema conradii, Heathland in the Annapolis Valley, Nova Scotia, 237

Catostomus commersoni, 21,221,295,360

Cattail, 16,72,221,403 Broad-leaved, 17

Common, 420

Cedar, Eastern White, 386

Northern White, 404

Western Red, 160,344

Celastrina echo, 249

Celery, Wild, 546

Centromerita bicolor, 260

Centromerus bicolor, 260

cornupalpis, 260

denticulatus, 260

furcatus, 260

latidens, 260

longibulbus, 260

persolutus, 260

sylvaticus, 260

Centropyxis, 410

impressa, 407

Cerastium alpinum, 277

arvense, 248

beeringianum, 277

semidecandrum, 248

Ceraticelus alticeps, 260

atriceps, 260

bulbosus, 260

fissiceps, 260

laetabilis, 260

laetus, 260

minutus, 260

similis, 260

Ceratinella brunnea, 260

ornatula, 260

parvula, 260

Ceratinopsis nigriceps, 261

nigripalpis, 261

Ceratophyllum demersum, 16,137,548

Cervus elaphus, 574

Ceryle alcyon, 69,293,379

Ceryle alcyon, During Fall Migration, Territorial Behavior in Belted Kingfishers, 293

Cestode, 324

Cetraria, 506 
Chaenorhinum minus, 438

Chalcoscirtus alpicola, 268

Chamaedaphne calyculata, 388

Chapleau, F., 359

Chara sp., 16,26

altaica, 32

aspera, 33

buckellii, 32

canescens, 26

contraria, 35

delicatula, 34

evoluta, 26

fragilis, 35

globularis, 34

hirsuta, 32

sibirica, 32

zeylanica, 30

Chara canescens, Charophytes of Insular Newfoundland II: Chara evoluta and, 26

Chara evoluta and Chara canescens, Charophytes of Insular Newfoundland II: 26

Charadrius montanus, 532 vociferus, 70,379

Charadrius montanus, Differential Parental Care by Adult Mountain Plovers, 532

Chatte de l'est, 360

Chaulk, K., S. Bondrup-Nielsen, and F. Harrington. Black Bear, Ursus americanus, Ecology on the Northeast Coast of Labrador, 164

Chen caerulescens, 212,483 caerulescens caerulescens, 497 rossi, 483

Chêne rouge, 129

Cheniseo sphagnicultor, 261

Chenopodium spp., 548 dessicatum, 277 leptophyllum, 283,437

Cherry, Black, 371

Chestnut, American, 343 Horse, 344

Chickadee, 121

Black-capped, 71,367

Boreal, 70,367

Chicken, Greater Prairie, 507,515

Chickweed, Field, 248

Chimaerid, 539

Chipmunk, 578

Eastern, 323

Chironomidae, 548

Chlidonias niger, 69,78

Chou puant, 129

Christie, K.S. and T.E. Reimchen. Post-Reproductive Pacific Salmon, Oncorhynchus spp., as a Major Nutrient Source for Large Aggregations of Gulls, Larus spp., 202

Chrysemys picta, 389

Chrysosplenium tetrandrum, 277

Chub, Creek, 295,351 Lake, 221,351

Chubbs, T.E. and F.R. Phillips. Evidence of Range Expansion of Eastern Coyotes, Canis latrans, in Labrador, 381

Chytonix sensilis, 12

Cicuta bulbifera, 388

Cinquefoil, Bipinnate, 431

Circus cyaneus, 69,400
Cirsium foliosum, 418

Cistothorus palustris, 69

CITES Control List 2005, 319

Cladina sp., 78,413

Cladonia, 78

$$
\text { stellaris, } 78
$$

Cladophora, 84

Clangula hyemalis, 181

Clangula hyemalis, Eider, Somateria spp., and Scoter, Melanitta spp., Distributions in Central Alaska Beaufort Sea Lagoons, 1999-2002, Long-tailed Duck, 181

Clark, H.O. Jr. Aggressive Behaviour Exhibited by a San Joaquin Kit Fox, Vulpes macrotis mutica, 134

Claytonia perfoliata, 248

Cleavers, 248,439

Clematis occidentalis, 418 occidentalis ssp. grosseserrata, 429

Clematis, Purple, 429

Clethrionomys spp., 122 gapperi, 43, 123,323,414 rutilus, 445

Cloudberry, 210,412

Club-moss, Mountain, 278 Sitka, 418

Clubiona abbotii, 257 bryantae, 257

canadensis, 257

furcata, 257

johnsoni, 258

kastoni, 258

kulczynskii, 258

mixta, 258

moesta, 258

norvegica, 258

obesa, 258

opeongo, 258

riparia, 258

trivialis, 258

Clubrush, Hudson Bay, 427 Soft-stem, 427

Clupea harengus, 216 pallasi, 112

Coccothraustes vespertinus, 71

Coccyzus erythropthalmus, 71

Cody, W.J., Reviews by, 150,463

Cody, W.J. and K.L. Reading. Additions and Range Extensions to the Vascular Plant Flora of the Continental Northwest Territories and Nunavut, Canada III, 276

Cody, W.J., B.A. Bennett, and P. Caswell. New Records of Vascular Plants in the Yukon Territory VII, 417

Coeloglossum viride ssp. bracteatum, 418

Colaptes auratus, 70,369,379

Collinsia clypiella, 262 palmeni, 262 parviflora, 248

Colpodium, 498 wrightii, 498

Comandra umbellata ssp. pallida, 418

Commandra, Pale, 428

Commorant, Double-crested, 69

Comptonia peregrina, 9,240

Consaul, L.L., L.J. Gillespie, and K.I. MacInnes. Addition to the Flora of Canada? A Specimen from the Arctic Archipelago, Northwest Territories Links Two Allopatric Species of Alkali Grass, Puccinellia, 497 
Contopus virens, 71

Cook, F.R., Reviews by, 141,145,148,154

Cooke, S.J., Review by, 147

Coontail, 16,137

Coot, American, 69

Coras montanus, 256

Coregonus huntsmani, 294

Coregonus huntsmani, from the Wild, First Record of Age 0+ Atlantic Whitefish, 294

Corema conradii, 237

Corema, 237

Corema conradii, Heathland in the Annapolis Valley, Nova Scotia, nvasive Scots Pine, Pinus sylvestris, Replacing Corema, 237

Corema, Corema conradii, Heathland in the Annapolis Valley, Nova Scotia, Invasive Scots Pine, Pinus sylvestris, Replacing, 237

Coreopsomela, 89

Coriarachne versicolor, 270

Corixidae, 548

Cormorant, Double-crested, 72

Cornicularia auranticeps, 266 cuspidata, 266

karpinskii, 266

minuta, 266

unicornis, 266

vigilax, 266

Cornus, 96

canadensis, 44,412

racemosa, 17

sericea

Corvus brachyrhynchos, 70,291,443 corax, 70,291,444

Corvus brachyrhynchos, Predation of a Bat by American Crows, 443

Corylus spp., 369 americana, 17,96 cornuta, 457

Cotton-grass, Slender, 426

Cottonwood, 17 Plains, 132

Cottus sp., 582

Coturnicops noveboracensis, 69

Couesius plumbeus, 221,351

Cougar, 574,580

Cougar, Puma concolor, in the Yukon, First Confirmation of, 580

Couleuvre à collier, 457 rayée, 458

Couleuvre à collier, Diadophis punctatus edwardsii, dans l'est du Québec, Extension de l'aire de distribution de la, 457

Coydog, 3

Coyote, 1,134,139,161,200,574,580,584

Eastern, 324,381

Coyote, Canis latrans, in New York, Chronology of Range Expansion of the, 1

Coyote, Canis latrans, Record Size Female, 139

Coyotes, Canis latrans, in Labrador, Evidence of Range Expansion of Eastern, 381

Cranberry, Dry-ground, 210 Mountain, 288

Crane, Sandhill, 69

Crapet-soleil, 360

Crappie, Black, 21
White, 21

Crataegus sp., 17

Crayfish, 17

Crematogaster cerasi, 39

Crepis nana, 277

Cress, Aleutian, 429

Saltwater, 429

Crossbill, Red, 121

White-winged, 70

Crotalus, 95

Crow, American, 70,291,443

Crowberry, 255

Black, 210

Crows, Corvus brachyrhynchos, Predation of a Bat by American, 443

Crustulina borealis, 269 sticta, 269

Cryphoeca montana, 259

Cryptogramma crispa var. acrostichoides, 277 crispa var. sitchensis, 418

Crystophora cristata, 382

Ctenium banksi, 269 boreale, 269

fuscum, 269 riparium, 269

Cuckoo, Black-billed, 71

Culaea inconstans, 221,360

Curlew, Long-billed, 490

Currier, C., 132

Cutgrass, Rice, 547

Cuthbert, F.J., 367

Cyanocitta cristata, 71,377

Cybaeopsis euopla, 256 tibialis, 256

Cybaeota calcarata, 258

Cyclopyxis, 403 acmodonta, 403 arcelloides, 409 crucistoma, 407 grospietschi, 407 impressa, 407 lobostoma, 408 stellata, 408 trilobata, 407 trilobata var. maxima, 407

Cyclopyxis acmodonta n. sp. and Arcella formosa n. sp.: Two New Species of Testate Rhizopods (Arcellinida, Protozoa) from Remnant Wetlands in Ontario, Canada, 403

Cyclosa conica, 257

Cynanchum rossicum, 525

Cynomys ludovicianus, 532

Cyperus erythrorhizos, 548 odoratus, 548

Cyprinella spiloptera, 21

Cyprinus carpio, 21,546

Cypripedium acaule, 240

Cystopteris fragilis, 276 montana, 277

Cytisus scoparius, 245

Dace, Longnose, 221

Northern Redbelly, 221

Pearl, 221

Dactylis glomerata, 552 
Daisy, Arctic-alpine, 435

Large-flowered, 435

Northern, 435

Subalpine, 435

Danaus plexippus, 525

Dandelion, 552

Rock, 436

Danthonia spicata, 240,277

Dard à ventre jaune, 361

Darimont, C.T., 192

Darner, Common Green, 234

Darter, 582

Blackside, 18

Iowa, 221

Johnny, 19

d'aster du Saint-Laurent, Symphyotrichum laurentianum, aux Îles-de-la-Madeleine, Protocole de suivi des populations, 556

Davies, C., 453

Davies, J.C., 64

Davis, H., 296

Death-camas, Meadow, 248

Deer, Mule, 296,574,580

White-tailed, 101,330,381,573,580,591

Deer, Odocoilus hemionus, and Other Mammals, Passage Through a Small Drainage Culvert by Mule, 296

Deer, Odocoileus virginianus, Moose, Alces alces, and Beaver, Castor canadensis, in Algonquin Park, Ontario, 1860-2004, Reconstructing Changes in Abundance of White-tailed, 330

de Lafontaine, G. Protocole de suivi des populations d'aster du Saint-Laurent, Symphyotrichum laurentianum, aux Îles-de-la-Madeleine, 556

Delphinapterus leucas, 214

Delphinus bairdii, 110

capensis, 110

delphis, 110 delphis bairdii, 110

Delphinus capensis, in Canadian Waters, First Records of Long-beaked Common Dolphins, 110

Dendroctonus ponderosae, 186

Dendroica caerulescens, 70

coronata, 70

magnolia, 70

palmarum, 69

pensylvanica, 71

petechia, 70

tigrina, 71

virens, 70

Dendryphantes nigromaculatus, 268

Dermacentor albipictus, 330

Dermatocarpon, 78 reticulatum, 78

Deschampsia flexuosa, 239

Descurainia sophioides, 277

Desmognathus fuscus, 105

Desmognathus fuscus, sur la rive nord du fleuve Saint-Laurent, au Québec, Premières mentions et répartition de la Salamandre sombre du Nord, 105

de Solla, S.R., 58

Desroches, J.-F., 129

Desroches, J.-F. et B. Roussel. Extension de l'aire de distribution de la Couleuvre à collier, Diadophis punctatus edwardsii, dans l'est du Québec, 457

Desroches, J.-F. et D. Pouliot. Premières mentions et répartition de la Salamandre sombre du Nord, Desmog- nathus fuscus, sur la rive nord du fleuve Saint-Laurent, au Québec, 105

Devine, A. and D.G. Smith. Caching Behavior by Wintering Northern Saw-Whet Owls, Aegolius acadicus, 578

Devries, J.H., 541

Diadophis punctatus edwardsii, 457

Diadophis punctatus edwardsii, dans l'est du Québec, Extension de l'aire de distribution de la Couleuvre à collier, 457

Dianthus barbatus, 438

Dichanthelium acuminatum ssp. fasciculatum, 240 depauperatum, 240

Dicrocoelium dentriticum, 324

Dicrostonyx groenlandicus, 124

Dictyna alaskae, 258

annulipes, 258

arundinacea, 258

bostoniensis, 258

brevitarsus, 258

minuta, 258

phylax, 258

Dicymbium elongatum, 261 nigrum, 261

Dietrichia hesperia, 261

Difflugia lobostoma var. impressa, 407

Dimelaena, 78 oreina, 78

Dinsmore, S.J. and F.L. Knopf. Differential Parental Care by Adult Mountain Plovers, Charadrius montanus, 532

Diphasiastrum sitchense, 417

Diplocentria bidentata, 261 corynetes, 264 rectangulata, 261

Diplocephalus cristatus, 261 cuneatus, 261 subrostratus, 261

Diplodia, 226

Dipoena nigra, 269

Dismodicus alticeps, 261 bifrons decemoculatus, 261 decemoculatus, 261

Dock, Golden, 429

Dodecatheon hendersonii, 248

Dog, Coyote-domestic, 3

Dogbane, Spreading, 433

Dogwood, 17,96

Dolichoderus plagiatus, 39

Dolichonyx oryzivorus, 70

Dolomedes, 255

fulviatronotatus, 255

scapularis, 255

striatus, 255

triton, 255

vittatus, 255

Dolphin, Bottlenose, 217

Dusky, 217

Long-beaked Common, 110

Short-beaked Common, 110

Dolphins, Delphinus capensis, in Canadian Waters, First Records of Long-beaked Common, 110

Dondale, C.D., 254

Donovan, D., 453

Dorosoma cepedianum, 21

Douglas-fir, 344

Douglasia ochotensis, 418

Dove, Mourning, 71,379 
Draba albertina, 418

cinerea, 418

crassifolia, 418

fladnizensis, 276

juvenalis, 277

lactea, 286

lonchocarpa var. vestita, 418

longipes, 285

nivalis, 276,418

oligosperma, 418

scotteri, 418

stenoloba, 418

stenopetala, 418

wahlenbergii, 276

Draba, Alaska, 430

Few-seeded, 430

Slender, 429

Snow, 430

Dragonfly (Odonata) Diversity as a Bioindicator of the Efficiency of Sewage Lagoons, A Potential for the Use of, 233

Drapetisca alteranda, 261

Drassodes neglectus, 258

Drepanocladus, 78

Drosera anglica, 418 rotundifolia, 388

Drum, Freshwater, 21

Drummond, D.R., 135

Dryas, 506

crenulata, 418

hookeriana, 418

integrifolia, 210

integrifolia ssp. crenulata, 418

octopetala ssp. hookeriana, 418

Dryocopus pileatus, 70,367,379

Dryopteris fragrans, 276 spinulosa, 570

Duck, 78,546

American Black, 69

Long-tailed, 181

Mallard, 69

Ring-necked, 69

Wood, 69,379,547

Duck, Clangula hyemalis, Eider, Somateria spp., and Scoter, Melanitta spp., Distributions in Central Alaska Beaufort Sea Lagoons, 1999-2002, Long-tailed, 181

Ducks During Fall Migration in a Prairie Pothole System, Heron Lake, Minnesota, Food Habits of Dabbling, 546

Dulichium arundinaceum, 390

Dumetella carolinensis, 70

Dunbrack, R. and R. Zielinski. Body Size Distribution and Frequency of Anthropogenic Injuries of Bluntnose Sixgill Sharks, Hexanchus griseus, at Flora Islets, British Columbia, 537

Dunlin, 379

Dwarf-Primrose, Arctic-Montane, 433

Echiniscus spitsbergensis, 587

Echinochloa crusgalli, 548

Editor's Report for Volume 118 (2004), 321

Eel, American, 294,351

Eelgrass, 16,28

Eider, 181

Common, 182

King, 182
Eider, Somateria spp., and Scoter, Melanitta spp., Distributions in Central Alaska Beaufort Sea Lagoons, 19992002, Long-tailed Duck, Clangula hyemalis, 181

Eidmanella pallida, 267

Eleocharis sp., 394,548

erythropoda, 439

obtusa, 394

Elk, 574

Elle, E., 245

Elm, 369

American, 96,370

Slippery, 370

Elymus canadensis, 277

junceus, 422

macrourus, 277

trachycaulus ssp. violaceus, 277

Emberiza citrinella, 120

Emblyna annulipes, 258 phylax, 258

Emery, R.B., 541

Empetrum, 255 nigrum, 210

Empidonax alnorum, 70

flaviventris, 69

minimus, 70

tyrannus, 70

Emydoidea blandingi, 389

Enallagma, 234

civile, 234

cyathigerum, 234

Encalypta sp., 80

Enoplognatha caricis, 269

intrepida, 269

marmorata, 269

ovata, 269

Entelecara abrupta, 266

exigua, 264

sombra, 261

Enteromorpha, 84

Eperigone bryantae, 261

contorta, 261

entomologica, 261

index, 261

maculata, 261

trilobata, 261

undulata, 261

Epilobium ciliatum, 394

coloratum, 394

davuricum, 276

hornemannii ssp. hornemannii, 418

latifolium $\mathrm{f}$. albiflorum, 276

palustre, 277

Épinette, 457

blanche, 458

noire, 457

Épinoche à cinq épines, 360

à trois épines, 360

Epitheca cynosura, 234

Eptesicus fuscus, 132

Equisetum palustre, 276

Equus cabalus, 574

Érable à épis, 457

à sucre, 129,457

argenté, 129

de Pennsylvanie, 129

Eremophila alpestris, 71 
Erethizon dorsatum, 125,159,177,453

Erethizon dorsatum, in Second-Growth Conifer Forests, The Influence of Thermal Protection on Winter Den Selection by Porcupines, 159

Eridantes utibilis, 261

Erigeron grandiflorus ssp. arcticus, 418 humilis, 418

peregrinus ssp. peregrinus, 418

philadelphicus, 394

pumilus, 418

uniflorus ssp. eriocephalus, 277,418

yukonensis, 418

Erigone aletris, 261

atra, 261

blaesa, 261

dentigera, 261

dentipalpis, 255

ephala, 261

mentasta, 262

whymperi, 262

Eriophorum angustifolium, 277

callitrix, 277

gracile, 418

triste, 277

vaginatum, 277

viridi-carinatum, 276

Ero canionis, 267

Erskine, A.J., Review by, 299

Erynnis propertius, 249

Erysimum pallasii, 276

Erythemis simplicicollis, 234

Erythronium oregonum, 248

Esox lucius, 21,359 masquinongy, 364

Esox lucius, dans le lac Ramsay, Parc de la Gatineau, Québec, et impact sur l'ichtyofaune, Charactéristiques d'une population introduit du Grand brochet, 359

Estrandia grandaeva, 262

Etheostoma sp., 582 exile, 221,361 nigrum, 21

Eulaira concava, 259 microtarsus, 262

Eupatorium maculatum, 394 perfoliatum, 389

Euphagus cyanocephalus, 69

Euphrasia stricta, 390

Eurycea bislineata, 106

Euthamia graminifolia, 388 graminifolia var. graminifolia, 290

Eutrema edwardsii, 277

Euxoa incallida, 12 scandens, 12

Evarcha hoyi, 268

Evers, D., 455

Ewins, P.J., 58

Fagus grandifolia, 38,129,332,378

Falco columbarius, 71,379

peregrinus, 70 sparverius, 71,379

Falcon, Peregrine, 70

Farnell, R., P.G. Hare, and D.R. Drummond. An Ancient Wolf, Canus lupus, Den and Associated Human Activity in the Southwestern Yukon Territory, 135

Felis concolor, 574
Fener, H.M., J.R. Ginsberg, E.W. Sanderson, and M.E. Gompper. Chronology of Range Expansion of the Coyote, Canis latrans, in New York, 1

Fern, Fragile, 278

Fragrant Cliff, 278

Holly, 419

Lady, 419

Mountain Bladder, 278

Sitka Parsley, 419

Sweet, 9

Wood, 570

Fescue, Alaska, 421

Alpine, 421

Little, 421

Festuca brachyphylla, 276,418

brevissima, 418

filiformis, 240

minutiflora, 418

richardsonii, 277

rubra ssp. richardsonii, 279

Finch, Purple, 70

Finney-Crawley, J.R., 323

Fir, Amabilis, 160

Balsam, 255,381,570

Subalpine, 186

Fisher, 120,177,194

Fivefinger, Marsh, 287

Fleabane, Shaggy, 435 Yukon, 435

Flicker, Northern, 70,369,379

Floricomus rostratus, 262

Flycatcher, Alder, 70

Great-crested, 367

Least, 70

Yellow-bellied, 69

Fontaine, A.J., 483

Ford, J.K.B. First Records of Long-beaked Common Dolphins, Delphinus capensis, in Canadian Waters, 110

Forget-me-not, Common, 248

Forktail, Eastern, 234

Formica aserva, 40

glacialis, 39

lasioides, 39

neogagates, 40

nepticula, 40

nitidiventris, 40

subaenescens, 40

subsericea, 40

$42^{\text {nd }}$ North American Moose Conference, 12-16, 2006 Baddeck, Nova Scotia, Canada, 320

Fowlie, A.R., 582

Fox, Arctic, 120,323

Red, 120,323

San Joaquin Kit, 134

Swift, 134,584

Fox, Vulpes macrotis mutica, Aggressive Behaviour Exhibited by a San Joaquin Kit, 134

Fox, Vulpes velox, Den Located Next to a Railroad Track in Northwestern Texas, Swift, 584

Foxtail, Meadow, 421

Fragaria virginiana, 394

Francoeur, A., 38

Fraxinus nigra, 457

Freeling, S.E., 219

Frêne noir, 457

Fritillaria affinis, 248 
Frog, Boreal Chorus, 291

Columbia Spotted, 291

Northern Leopard, 389

Pickerel, 578

Froglog: Newsletter of the Declining Amphibian Populations Task Force, 156

Fulica americana, 69

Fundulus diaphanus, 21,28,295

Gadwall, 547

Galium aparine, 248,439 tinctorium, 394

trifidum, 276

triflorum, 418

Gallant, J.J., 355

Gallinago delicata, 69

Gambelia, 95

Gar, Longnose, 21

Gasterosteus aculeatus, 28,360

Gaultheria hispidula, 44 procumbens, 240

Gavia immer, 455

Gavia immer, Nest Attendance Patterns Recorded by Remote Video Camera, Common Loon, 455

Gawn, M., Review by, 604

Gaylussaccia baccata, 240

Geese, Branta hutchinsii, in the Eastern Canadian Arctic, Breeding and Non-Breeding Range of Canada, Branta canadensis, and Cackling, 483

Gentiana affinis, 277

Geomys spp. 175

Geopyxella sp., 408

Geothlypis trichas, 69

Geranium erianthum, 418 molle, 248

Geranium, Dovefoot, 248 Northern, 432

Ghelna canadensis, 268

Gilbert, B.K., 449

Gillespie, L.J., 497

Ginsberg, J.R., 1

Glaucomys sabrinicus, 344 sabrinus, 591 volans, 374

Gleason, J.S., R.A. Hoffman, and J.M. Wendland. Beavers, Castor canadensis, Feeding on Salmon Carcasses: Opportunistic Use of a Seasonally Superabundant Food Source, 591

Glenostictia, 6

Glycine max, 547

Glyphesis scopulifer, 262

Gnaphosa brumalis, 258 microps, 258 muscorum, 258 parvula, 258

Gnathonaroides pedalis, 262

Goat, Mountain, 197,574

Goby, Round, 18,582

Goby, Neogobius melanostomus, in the St. Lawrence River at Cornwall, Ontario, First Occurrence of the Round, 582

Gold, Spring, 248

Goldeneye, Common, 69

Goldfinch, American, 71

Goldfish, 18
Gómez-Zurita, J. New Distribution Records and Biogeography of Calligrapha Species (Leaf Beetles), in North America (Coleoptera: Chrysomelidae, Chrysomelinae), 88

Gompper, M.E., 1

Gonatium crassipalpum, 262

Gonzales, E.K. The Distribution and Habitat Selection of Introduced Eastern Grey Squirrels, Sciurus carolinensis, in British Columbia, 343

Goodale, W., L. Attix, and D. Evers. Common Loon, Gavia immer, Nest Attendance Patterns Recorded by Remote Video Camera, 455

Goodwin, C.E., Reviews by, 144,600

Goose, Cackling, 483

Canada, 69,379,483,549

Lesser Snow, 497

Ross's, 483

Snow, 212,483

Gooseberry, 369

Goosefoot, Narrow-leaved, 283,438

Gopher, Northern Pocket, 175,551 Valley Pocket, 551

Gophers, Thomomys talpoides, in Central Alberta Alfalfa Fields, Body Weights of Adult and Juvenile Northern Pocket, 551

Gophers, Thomomys talpoides, Long-tailed Weasel, Mustela frenata, Movements and Diggings in Alfalfa Fields Inhabited by Northern Pocket, 175

Gosse, J.W. and B.J. Hearn. Seasonal Diets of Newfoundland Martens, Martes americana atrata, 43

Grackle, Common, 70

Gramma, Blue 220

Grammonota angusta, 262

capitata, 262

gigas, 262

maritima, 262

pictilis, 262

vittata, 262

Grand brochet, 359

Grand brochet, Esox lucius, dans le lac Ramsay, Parc de la Gatineau, Québec, et impact sur l'ichtyofaune, Charactéristiques d'une population introduit du, 359

Graphicallo, 89

Grasby, S.E., 82

Grass, Alkali, 497

Alkali Cord, 280

Alpine Blue, 280

American Lyme, 421

Common Panic, 439

Ice, 422

Kentucky Blue, 245

Needle-and-thread, 220

Orchard, 552

Reed Canary, 422

Turtle, 114

Western Wheat, 220

Widgeon, 114

Grass, Puccinellia, Addition to the Flora of Canada? A Specimen from the Arctic Archipelago, Northwest Territories Links Two Allopatric Species of Alkali, 497

Grass-of-Parnassus, Fringed, 431

Greasewood, 220

Grebe, Horned, 69 Pied-billed, 69

Grosbeak, Evening, 71 Pine, 121 
Rose-breasted, 71

Grouse, Black, 120

Hazel, 120

Northern Sharp-tailed, 507,515

Prairie, 507,515

Prairie Sharp-tailed, 507,515

Ruffed, 44,71,379

Sharp-tailed, 507

Willow, 120

Grouse", Tympanuchus cupido $\times$ phasianellus, Hybridization on Manitoulin Island, Ontario, "Prairie, 507

Grouse", Tympanuchus cupido $\times$ phasianellus, of Manitoulin Island, Ontario, Plumage and Internal Morphology of the "Prairie, 515

Grus canadensis, 69

Gull, 202

Bonaparte's, 203

California, 203

Glaucous-winged, 203

Mew, 203

Ring-billed, 69

Thayer's, 203

Gulls, Larus spp., Post-Reproductive Pacific Salmon, Oncorhynchus spp., as a Major Nutrient Source for Large Aggregations of, 202

Gulo gulo, 120

Gyrinophilus porphyriticus, 106

Habenaria tridentata, 388

Hahnia cinerea, 259 glacialis, 259

Hake, Pacific, 112

Halenia deflexa, 438

Halichoerus grypus, 355

Halictus sp., 249

Halorates alascensis, 262

holmgrenii, 262

palmeni, 262

pertinens, 262

Hamas, M.J. Territorial Behavior in Belted Kingfishers, Ceryle alcyon, During Fall Migration, 293

Hammer, U.T., 114

Haplodrassus hiemalis, 258 signifer, 258

Hare, Arctic, 323 European, 122

Snowshoe, 43,121,197,323,395,569

Hare, Lepus americanus, on Insular Newfoundland During a Cyclical Peak, Analysis of the Parasites of a Midwinter Population of the Snowshoe, 323

Hare, P.G., 135

Harebell, 434

Hares, Lepus americanus, on Kent Island, New Brunswick, Tree Recruitment Limitation by Introduced Snowshoe, 569

Harrier, Northern, 69,400

Harrimanella stellariana, 418

Harrington, F., 164

Harvey-Clark, C.J., J.J. Gallant, and J.H. Batt. Vision and its Relationship to Novel Behaviour in St. Lawrence River Greenland Sharks, Somniosus microcephalus, 355

Hasselman, D.J., P. Longue, and R.G. Bradford. First Record of Age 0+ Atlantic Whitefish, Coregonus huntsmani, from the Wild, 294
Hawk, Broad-winged, 71,377

Cooper's, 379

Ferruginous, 490

Red-shouldered, 377

Sharp-shinned, 377

Hawkweed, Slender, 435

Hawthorn, 17

Hazel, 369

Hazelnut, 17

American, 96

Hearn, B.J., 43

Hedysarum alpinum, 277

boreale ssp. mackenzii, 277

mackenzii, 287

Helophora insignis, 262

ontariensis, 259

Hemidactylium scutatum, 129

Hemidactylium scutatum, à Québec, Québec: limite nord-est de l'espèce sur la rive nord du fleuve Saint-Laurent, Découverte de la Salamandre à quatre orteils, 129

Hemlock, 38,332

Eastern, 386

Mountain, 291

Western, 160,197,344

Hen, Heath, 516

Hendricks, P., J. Johnson, S. Lenard, and C. Currier. Use of a Bridge for Day Roosting by the Hoary Bat, Lasiurus cinereus, 132

Heron, Great Blue, 69,379

Herring, 203

Pacific, 112,216

Hêtre à grandes feuilles, 129

Hexanchus griseus, 537

Hexanchus griseus, at Flora Islets, British Columbia, Body Size Distribution and Frequency of Anthropogenic Injuries of Bluntnose Sixgill Sharks, 537

Hickey, M.B.C. and A.R. Fowlie. First Occurrence of the Round Goby, Neogobius melanostomus, in the St. Lawrence River at Cornwall, Ontario, 582

Hickory, 343,369

Hieracium gracile, 418 pilosella, 240

Hierochloe hirta ssp. arctica, 277 odorata, 280

Hilaira algida, 265

aquilonia, 262

canaliculata, 262

dubia, 264

herniosa, 262

mentasta, 262

Hippuris vulgaris, 28

Hirundo rustica, 70

Hoffman, R.A., 591

Holyan, J., D.K. Boyd, C.M. Mack, and D.H. Pletscher. Longevity and Productivity of Three Wolves, Canis lupus, in the Wild, 446

Honeybee, 250

Hood, D.J. and R.F. Stocek. The Fathead Minnow, Pimephales promelas, in New Brunswick, 351

Hordeum vulgare, 438

Horse, 574

Horsetail, Marsh, 278

Houston, C.S. Long-eared Owls, Asio otus: A Review of North American Banding, 395

Houston, C.S., Reviews by, 595,596,598,606,607,608 
Howerter, D.W., 541

Hudsonia ericoides, 240

Huettmann, F., Reviews by, 142,151,302,313,314,315,467,601

Hulteniella integrifolia, 506

Hummingbird, Ruby-throated, 70,379

Hybauchenidium gibbosum, 262

Hybocoptus denticulatus, 262

Hybognathus spp., 219

argyritis, 219

hankinsoni, 219,360

nuchalis, 219

placitus, 219

regius, 219

Hybognathus placitus, in Canada, First Record of the Plains Minnow, 219

Hydatigera taeniaeformis, 324

Hydrocotyle americana, 394

Hydrolagus colliei, 539

Hylocichla mustelina, 70

Hylocomium splendens, 404

Hypericum mutilum, 394 perforatum, 14,240

Hypomma marxii, 262

Hypsibius, 586 cf. convergens, 587

Hypsosinga pygmaea, 257 rubens, 257

Hyptiotes gertschi, 270

Ictalurus punctatus, 21

Icterus galbula, 70

Ictiobus cyprinellus, 21

If du Canada, 457

Impatiens capensis, 394

Iris setosa ssp. interior, 418 versicolor, 388

Ischnura verticalis, 234

Islandiana alata, 262 flaveola, 262 holmi, 262 princeps, 263

Isohypsibius $c f$. tuberculatus, 587

Iviella sp., 258

Jacob's Ladder, Showy, 433

Jaeger, Parasitic, 211

Jay, Blue, 71,377 Gray, 70,101,291

Jays, Perisoreus canadensis: Adaptations to Boreal Winters, Weight-carrying Ability and Caching Behavior of Gray, 101

Jays, Perisoreus canadensis, on Long-toed Salamanders, Ambystoma macrodactylum, in the Oregon Cascade Range, Apparent Predation by Gray, 291

John, R., Reviews by, 141,146,149,299,462,594,599

Johnson, J., 132

Johnson, S.R., 181

Joynt, B.L., 541

Juglans nigra, 348

Junco, 578

Juncus albescens, 282

arcticus, 276

brevicaudatus, 388

bufonius, 418

canadensis, 394 castaneus ssp. castaneus, 276

filiformis, 418

stygius ssp. americanus, 277

triglumis ssp. albescens, 277

Jung, T.S. and B.G. Slough. Mortality of Little Brown Bats, Myotis lucifugus, in a Rodent Trap in the Boreal Forest, 589

Jung, T.S. and K.S. O'Donovan. Mortality of Deer Mice, Peromyscus maniculatus, in Wire Mesh Live-Traps: A Cautionary Note, 445

Jung, T.S. and P.J. Merchant. First Confirmation of Cougar, Puma concolor, in the Yukon, 580

Jung, T.S., K.S. O'Donovan, and T. Powell. Long-distance Movement of a Dispersing Deer Mouse, Peromyscus maniculatus, in the Boreal Forest, 451

Juniper, 78

Ground, 278

Juniperus, 78

communis, 240,277

Kaestneria pullata, 263 rufula, 263

Kalmia angustifolia, 240,381

Kariz, R.M., 186

Kelly, E., Review by, 311

Kestrel, American, 71,379

Kevan, P.G., 48

Killdeer, 70,379

Killifish, Banded, 18,28,294

Kingbird, Eastern, 70

Kingfisher, Belted, 69,293,379

Kingfishers, Ceryle alcyon, During Fall Migration, Territorial Behavior in Belted, 293

Kinglet, Golden-crowned, 579 Ruby-crowned, 70

Knopf, F.L., 532

Knotweed, Striate, 428

Krawchuk, A., K.W. Larsen, R.D. Weir, and H. Davis. Passage Through a Small Drainage Culvert by Mule Deer, Odocoilus hemionus, and Other Mammals, 296

Kuker, K.J., J.A. Thomson, and U. Tscherter. Novel Surface Feeding Tactics of Minke Whales, Balaenoptera acutorostrata, in the Saguenay-St. Lawrence National Marine Park, 214

Kurczewski, F.E. and H.F. Boyle. Nesting Behavior, Ecology, Seasonal and Geographic Distribution of the Sand Wasp, Stictiella emarginata (Hymenoptera: Sphecidae)*, 6

Kuzyk, G.W., C. Rohner, and F.K.A. Schmiegelow. Travel Rates of Wolves, Canis lupus, in Relation to Ungulate Kill Sites in Westcentral Alberta, 573

Labidesthes sicculus, 21

Labrador Tea, 288,381,412 Dwarf, 210

Labrador, A Range Extension for the Rock Vole, Microtus chrotorrhinus, in, 412

Labrador, Black Bear, Ursus americanus, Ecology on the Northeast Coast of, 164

Labrador, Evidence of Range Expansion of Eastern Coyotes, Canis latrans, in, 381

Lacinipolia vicina, 12

Lady's-thumb, 428

Lady's-smock, 285

Lagenorhynchus obscurus, 217 
Lagopus lagopus, 44,120

Lagotis glauca, 277 glauca ssp. minor, 288

Lambdina fiscellaria, 324

Lance, Sand, 216

Lanius sp., 102,578 ludovicianus, 490

Lansing, S.W. A Range Extension for the Rock Vole, Microtus chrotorrhinus, in Labrador, 412

Lapierre, K. and Coad, B.W, Review by, 301

Larinioides cornutus, 257 patagiatus, 257 sclopetarius, 257

Larix decidua, 255 laricina, 404,574

Lark, Horned, 71

Larsen, K.W., 296

Larus spp., 202 argentatus, 203 californicus, 203 canus, 203 delawarensis, 69 glaucescens, 203 philadelphia, 203 thayeri, 203

Larus spp., Post-Reproductive Pacific Salmon, Oncorhynchus spp., as a Major Nutrient Source for Large Aggregations of Gulls, 202

Lasallia, 78 pensylvanica, 78

Lasiurus cinereus, 132

Lasiurus cinereus, Use of a Bridge for Day Roosting by the Hoary Bat, 132

Lasius alienus, 40 nearcticus, 40 neoniger, 40 niger, 40 pallitarsis, 40 umbratus, 40

Lassioglossum sp., 249

Lathyrus sphaericus, 248

Lauff, R., Reviews by, 144,462

Laurel, Sheep, 381

Lavallée, B.F., 359

Ledum decumbens, 210 groenlandicum, 276,381

Leersia oryzoides, 547

Lefevre, K.L. Predation of a Bat by American Crows, Corvus brachyrhynchos, 443

Lemming, Collared, 124

Lenard, S., 132

Lepisosteus osseus, 21

Lepomis cyanellus, 21 gibbosus, 21,360 humilis, 21 macrochirus, 21 megalotis, 21

Leproloma vouauxii, 78

Leptarrhena pyrolifolia, 418

Lepthyphantes spp., 263 alpinus, 263

bihamatus, 263

calcaratus, 263

complicatus, 263

cracens, 263 duplicatus, 263

intricatus, 263

leprosus, 263

nigriventris, 263

subalpinus, 263

tenuis, 263

triramus, 263

turbatrix, 263

umbraticola, 263

washingtoni, 263

zebra, 263

Leptothorax ambiguus, 39

canadensis, 39

ergatogyneus, 38

longispinosus, 39

Lepus americanus, 43,121,197,323,395,569

arcticus, 323

europaeus, 122

Lepus americanus, on Insular Newfoundland During a Cyclical Peak, Analysis of the Parasites of a Mid-winter Population of the Snowshoe Hare, 323

Lepus americanus, on Kent Island, New Brunswick, Tree Recruitment Limitation by Introduced Snowshoe Hares, 569

Leslie, J.K. and C.A. Timmins. Environment and Distribution of Age 0 Fishes in River Canard, a Lowland Ontario River, 16

Lestes disjunctus, 234

Lethocerus sp., 292

Leucorrhinia spp., 234 intacta, 234 proxima, 234

Leymus mollis ssp. mollis, 417

Libellula pulchella, 234 quadrimaculata, 234

Lichen, 413

Lichter, J., 569

Lily, Bullhead, 429

Chocolate, 248

White Fawn, 248

Limosella aquatica, 277

Linaria vulgaris, 14

Linnaea borealis var. americana, 277

Linyphia marginata, 264 waldea, 264

Liquorice-root, 287

Lithophragma parviflorum, 248

Little Nightmare, Eschscholtz', 429

Lizard, 95

Lobelia inflata 394

Locky, D.A., J.C. Davies and B.G. Warner. Effects of Wetland Creation on Breeding Season Bird Use in Boreal Eastern Ontario, 64

Locoweed, Blackish, 432

Logperch, 21

Lolium spp., 548

Lomatium utriculatum, 248

Londry, K.L., P.H. Badiou, and S.E. Grasby. Identification of a Marine Green Alga Percursaria percursa from Hypersaline Springs in the Middle of the North American Continent, 82

Longspur, Lapland, 208

Longspur, Calcarius lapponicus, Nests at Cape Churchill, Manitoba, Microhabitat Characteristics of Lapland, 208 
Longue, P., 294

Lonicera involucrata, 418

Lontra canadensis, 459

Lontra canadensis) Contribute to Fall Scent Marking?, Do Juvenile Nearctic River Otters, 459

Loon, Common, 455

Loon, Gavia immer, Nest Attendance Patterns Recorded by Remote Video Camera, Common, 455

Looper, Hemlock, 324

Loosestrife, Purple, 18

Lophodytes cucullatus, 69

Lophomma sylvaticum, 263

Lotus, American, 17

Lousewort, Lapland, 289

Loxia curvirostra, 121 leucoptera, 70

Luetkea pectinata, 418

Lumsden, H.G. Plumage and Internal Morphology of the "Prairie Grouse", Tympanuchus cupido $\times$ phasianellus, of Manitoulin Island, Ontario, 515

Lumsden, H.G. "Prairie Grouse", Tympanuchus cupido $\times$ phasianellus, Hybridization on Manitoulin Island, Ontario, 507

Lupine, Nootka, 432

Lupinus arcticus, 277 nootkatensis, 418

Lutra canadensis, 323 lutra, 459

Luxilus cornutus, 21,361

Luzula piperi, 418 spicata, 418 wahlenbergii, 277

Lycopodium sabinifolium ssp. sitchense, 418 selago, 277 sitchense, 417

Lycopus uniflorus, 277,388

Lynx canadensis, 323

Lynx, 323

Lysimachia thyrsifolia, 389

Lythrum salicaria, 18

MacInnes, K.I., 497

Mack, C.M., 446

MacPherson, A., Review by, 147

Macrobiotus cf. areolatus, 587 cf. hufelandi, 586

cf. islandicus, 587

cf. richtersi, 586

Madsen, J.D., 546

Madtom, Tadpole, 18

Maianthemum canadense, 240

Mallard, 379,541,546

Mallards, Anas platyrhynchos, Use of Radio-Telemetry to Test for Investigator Effects on Nesting, 541

Mallory, M.L., A.J. Fontaine, and H. Boyd. Breeding and Non-Breeding Range of Canada, Branta canadensis, and Cackling Geese, Branta hutchinsii, in the Eastern Canadian Arctic, 483

Mallotus villosus, 216,355

Mallow, Dwarf, 439

Malva neglecta, 439 rotundifolia, 438

Manitoba, Microhabitat Characteristics of Lapland Longspur, Calcarius lapponicus, Nests at Cape Churchill, 208
Mann, H. and E.M.V. Nambudiri. Charophytes of Insular Newfoundland II: Chara evoluta and Chara canescens, 26

Maple, Manitoba, 220

Red, 17,386

Sugar, 38,332,378,386

Mare's-tail, 28

Margariscus margarita, 221,360

Marine Turtle Newsletter, 156,319,473,613

Maro amplus, 263 nearcticus, 263

Marsh, J., 76

Marsh-marigold, 285 Floating, 285

Marten, 124

American, 43

Newfoundland, 43

Pine, 121,194,323

Martens, Martes americana atrata, Seasonal Diets of Newfoundland, 43

Martes americana, 43,124,194,323

americana atrata, 43

martes, 121

pennanti, 120,177,194

Martes americana atrata, Seasonal Diets of Newfoundland Martens, 43

Martin, P.A., S.R. de Solla, P.J. Ewins, and M.E. Barker. Productivity of Osprey, Pandion haliaetus, Nesting on Natural and Artificial Structures in the Kawartha Lakes, Ontario, 1991-2001, 58

Mary, Blue-eyed, 248

Maskinongé, 364

Maso sundevallii, 263

Matricaria ambigua, 277

McCarthy, J., Reviews by, 153,308,316,601

McGee, B.K., 584

McGrath, M., 323

McKague, C.I. and N. Cappuccino. Response of Pale Swallow-wort, Vincetoxicum rossicum, following Aboveground Tissue Loss: Implications for the Timing of Mechanical Control, 525

McMillan, B.R., 546

McNay, M.E. and P.W. Mooney. Attempted Predation of a Child by a Gray Wolf, Canis lupus, near Icy Bay, Alaska, 197

Meadow-foxtail, Water, 421

Meadowlark, Eastern, 71

Mecynargus paetulus, 263

Medicago spp., 175,551

Megachile sp., 250 rotundata, 53

Megaptera novaeangliae, 214

Megascops asio, 578

Meioneta sp. rurestris, 259

Melampyrum lineare, 240

Melandrium apetalum ssp. arcticum, 284

Melanelia, 78

disjuncta, 78

sorediata, 78

stygia, 78

Melanerpes carolinus, 369 erythrocephalus, 369

Melanitta spp., 181

fusca, 182 
nigra, 182

perspicillata, 182

Melanitta spp., Distributions in Central Alaska Beaufort Sea Lagoons, 1999-2002, Long-tailed Duck, Clangula hyemalis, Eider, Somateria spp., and Scoter, 181

Melospiza georgiana, 69

lincolnii, 70

melodia, 70,120

melodius, 578

Méné à nageoires rouges, 361 laiton, 360

Menyanthes trifoliata, 418

Mephitis mephitis, 454,584

Merchant, P.J., 580

Merganser, Common, 69 Hooded, 69

Mergus merganser, 69

Merlin, 71,379

Merluccius productus, 112

Merodon equestris, 249

Meta menardi, 268 ovalis, 268

Metaphidippus flavipedes, 268 montanus, 268

Metasyrphus, 250

Metepeira palustris, 257

Metridium senile, 355

Meunier noir, 360

Micaria aenea, 258

constricta, 259

longispina, 259

pulicaria, 259

rossica, 259

Mice, Peromyscus maniculatus, in Wire Mesh Live-Traps: A Cautionary Note, Mortality of Deer, 445

Micrargus longitarsus, 263

Microlinyphia mandibulata, 263

Micropterus dolomieu, 21,295,353,363 salmoides, 21,363

Microstictia, 6

Microtus spp., 122,395 chrotorrhinus, 412

chrotorrhinus chrotorrhinus, 412

chrotorrhinus ravus, 412

longicaudus, 445

pennslyvanicus, $43,323,414,446$ xanthognathus, 446

Microtus chrotorrhinus, in Labrador, A Range Extension for the Rock Vole, 412

Milfoil, Eurasian, 16

Milk-vetch, Alpine, 431

Milkweed, 525

Milnesium tardigradum, 586

Miner's-lettuce, 248

Mink, 175

Minnesota, Food Habits of Dabbling Ducks During Fall Migration in a Prairie Pothole System, Heron Lake, 546

Minnow, Bluntnose, 18

Brassy, 219

Eastern Silvery, 219

Fathead, 21,221,351

Mississippi Silvery, 219

Plains, 219

Pugnose, 18

Silvery, 219
Western Silvery, 219

Minnow, Hybognathus placitus, in Canada, First Record of the Plains, 219

Minnow, Pimephales promelas, in New Brunswick, The Fathead, 351

Minuartia biflora, 276

dawsonensis, 418

rossii, 276

rubella, 277

yukonensis, 277

Minyriolus castaneus, 266

Misumena vatia, 270

Mniotilta varia, 70

Moneses uniflora, 276

Monotropa hypopithys, 240

Montia linearis, 248

Montia, Narrow-leaved, 248

Mooney, P.W., 197

Moose, 43,50,136,168,186,194,197,323,330,381,574

Moose, Alces alces, and Beaver, Castor canadensis, in Algonquin Park, Ontario, 1860-2004, Reconstructing Changes in Abundance of White-tailed Deer, Odocoileus virginianus, 330

Moose, Alces alces, in Central Interior British Columbia, Winter Habitat Use by, 186

Morone americana, 21,295

chrysops, 21

Mosgovoyia pectinata, 324

Moss, 210,413,586

Mountain-avens, White, 210

Mountain-heather, Alaskan, 433

Mountain-parsley, 278

Mouse, 589,591

Cotton, 446

Deer, 194,323,414,445,451

Grasshopper, 95

House, 446,578

Jumping, 578

White-footed, 446,452,578

Woodland, 578

Mouse, Peromyscus maniculatus, in the Boreal Forest, Longdistance Movement of a Dispersing Deer, 451

Mouse-ear, Little, 248

Moxostoma sp., 21 macrolepidotum, 21

Mudminnow, Central, 19

Mudwort, 288 Aleutian, 435

Muhlenbergia glomerata, 277

Muldoon, F., 453

Mulet à cornes, 361 perlé, 360

Multiceps sp., 324 serialis, 324

Murray, M.P., C.A. Pear, and R.B. Bury. Apparent Predation by Gray Jays, Perisoreus canadensis, on Long-toed Salamanders, Ambystoma macrodactylum, in the Oregon Cascade Range, 291

Mus domesticus, 446 musculus, 578

Museau noir, 361

Muskox, 168

Muskrat, 570,591

Mustela erminea, 175,323

frenata, 175

vison, 175 
Mustela frenata, Movements and Diggings in Alfalfa Fields Inhabited by Northern Pocket Gophers, Thomomys talpoides, Long-tailed Weasel, 175

Myiarchus crinitus, 367

Myosotis discolor, 248

Myotis lucifugus, 132,444,589

Myotis, Little Brown, 132

Myotis lucifugus, in a Rodent Trap in the Boreal Forest, Mortality of Little Brown Bats, 589

Myriophyllum sp., 16 alterniflorum, 277 verticillatum, 418

Myrmecina americana, 39

Myrmica detritinodis, 39

emeryana, 39

fracticornis, 39

pinetorum, 39

punctiventris, 39

sculptilis, 39

Mythoplastoides exiguus, 264

Najas flexilis, 276,417

Nambudiri, E.M.V., 26

Neave, P., 453

Nedra ramosula, 12

Nelumbo lutea, 17

Nematode, 324

Némopanthe mucroné, 129

Nemopanthus mucronatus, 129

Neoantistea agilis, 259 magna, 259 riparia radula, 259

Neogobius melanostomus, 21,582

Neogobius melanostomus, in the St. Lawrence River at Cornwall, Ontario, First Occurrence of the Round Goby, 582

Neon nellii, 268

Neottiura bimaculata, 269

Neriene clathrata, 264 radiata, 264

Nero, R.W. Short-tailed Shrew, Blarina brevicauda, Apparently Killed by Red Squirrel, Tamiasciurus hudsonicus, 456

Nesticus pallida, 267

Nevin, O.T. and B.K. Gilbert. Observations of Autumn Courtship and Breeding in Brown Bears, Ursus arctos, from Coastal British Columbia, 449

New Brunswick, The Fathead Minnow, Pimephales promelas, in, 351

New Brunswick, Tree Recruitment Limitation by Introduced Snowshoe Hares, Lepus americanus, on Kent Island, 569

Newfoundland, An Annotated Checklist of the Spiders of, 254

Newfoundland During a Cyclical Peak, Analysis of the Parasites of a Mid-winter Population of the Snowshoe Hare, Lepus americanus, on Insular, 323

Newfoundland Martens, Martes americana atrata, Seasonal Diets of, 43

Newfoundland II: Chara evoluta and Chara canescens, Charophytes of Insular, 26

New York, Chronology of Range Expansion of the Coyote, Canis latrans, in, 1

Nicholls, K.H. Cyclopyxis acmodonta n. sp. and Arcella formosa n. sp.: Two New Species of Testate Rhizopods
(Arcellinida, Protozoa) from Remnant Wetlands in Ontario, Canada, 403

Nicholson, K.L., B.K. McGee, and W.B. Ballard. Swift Fox, Vulpes velox, Den Located Next to a Railroad Track

Nitella, 26 in Northwestern Texas, 584

confervacea, 35

flexilis, 35

opaca, 35

Noel, L.E., S.R. Johnson, and G.M. O'Doherty. Long-tailed Duck, Clangula hyemalis, Eider, Somateria spp., and Scoter, Melanitta spp., Distributions in Central Alaska Beaufort Sea Lagoons, 1999-2002, 181

Noisetier à long bec, 457

North America (Coleoptera: Chrysomelidae, Chrysomelinae), New Distribution Records and Biogeography of $\mathrm{Cal}$ ligrapha Species (Leaf Beetles), in, 88

North American Banding, Long-eared Owls, Asio otus: A Review of, 395

North American Continent, Identification of a Marine Green Alga Percursaria percursa from Hypersaline Springs in the Middle of the, 82

Northwest Territories and Nunavut, Canada III, Additions and Range Extensions to the Vascular Plant Flora of the Continental, 276

Northwest Territories from the Great Slave Lake Area, Additions to the Flora of the Continental, 437

Northwest Territories Links Two Allopatric Species of Alkali Grass, Puccinellia, Addition to the Flora of Canada? A Specimen from the Arctic Archipelago, 497

Notemigonus crysoleucas, 21,295,361

Notropis anogenus, 21

atherinoides, 21

heterolepis, 361

hudsonius, 21

stramineus, 21

volucellus, 21

Noturus gyrinus, 21

Nova Scotia, Invasive Scots Pine, Pinus sylvestris, Replacing Corema, Corema conradii, Heathland in the Annapolis Valley, 237

Nucifraga columbiana, 291

Numenius americanus, 490

Nunavut, Canada III, Additions and Range Extensions to the Vascular Plant Flora of the Continental Northwest Territories and, 276

Nuphar variegatum, 388,418

Nutcracker, Clark's, 291

Nuthatch, Red-breasted, 71,367 White-breasted, 367

Nymph, Wavy Water, 279,420

Nymphaea tetragona ssp. leibergii, 277

O'Doherty, G.M., 181

O’Donovan, K.S., 445,451

O’Neill, J., Reviews by, 463,464

Oak, 343,368

Bur, 348

Garry, 245,344

Red, 38,348,386

Scrub, 9

Oak Forests, Woodpecker Nest Tree Characteristics in Upper Midwestern, 367

Oak, Quercus garryana, Ecosystem of British Columbia, Insect Visitation to Wildflowers in the Endangered Garry, 245 
Oatgrass, Poverty, 279

Oats, 421

Obbard, M., 48

Obeliscoides cuniculi, 324

Oceanodroma leucorhoa, 570

Odocoileus heminous, 296,574,580 virginianus, 101,330,381,573,580,591

Odocoilus hemionus, and Other Mammals, Passage Through a Small Drainage Culvert by Mule Deer, 296

Odocoileus virginianus, Moose, Alces alces, and Beaver, Castor canadensis, in Algonquin Park, Ontario, 1860-2004, Reconstructing Changes in Abundance of White-tailed Deer, 330

Oedothorax montiferus, 266 trilobatus, 264

Olson, R.W., J.K. Schmutz, and U.T. Hammer. Occurrence, Composition and Formation of Ruppia, Widgeon Grass, balls in Saskatchewan Lakes, 114

Olson, Z.H., S.S. Stevens, and T.L. Serfass. Do Juvenile Nearctic River Otters (Lontra canadensis) Contribute to Fall Scent Marking? 459

Ommatokoita elongata, 355

Oncorhynchus spp., 202

gorbuscha, 202

keta, 202

kisutch, 197

tshawytscha, 591

Oncorhynchus spp., as a Major Nutrient Source for Large Aggregations of Gulls, Larus spp., Post-Reproductive Pacific Salmon, 202

Ondatra zibethicus, 570,591

Ontario: An Addition to the Flora of Canada, Papillate Watermeal, Wolffia brasiliensis, in Eastern, 137

Ontario, Canada, Cyclopyxis acmodonta n. sp. and Arcella formosa $\mathrm{n}$. sp.: Two New Species of Testate Rhizopods (Arcellinida, Protozoa) from Remnant Wetlands in, 403

Ontario, Effects of Wetland Creation on Breeding Season Bird Use in Boreal Eastern, 64

Ontario, First Occurrence of the Round Goby, Neogobius melanostomus, in the St. Lawrence River at Cornwall, 582

Ontario, Identification and Status of the Introduced Black Pine, Pinus nigra, and Mugo Pine, Pinus mugo, in, 224

Ontario, Movements of Two Rabid Raccoons, Procyon lotor, in Eastern, 453

Ontario Natural Heritage Information Centre Science and Information Newsletter, 319

Ontario, 1860-2004, Reconstructing Changes in Abundance of White-tailed Deer, Odocoileus virginianus, Moose, Alces alces, and Beaver, Castor canadensis, in Algonquin Park, 330

Ontario, Plumage and Internal Morphology of the "Prairie Grouse", Tympanuchus cupido $\times$ phasianellus, of Manitoulin Island, 515

Ontario, "Prairie Grouse", Tympanuchus cupido $\times$ phasianellus, Hybridization on Manitoulin Island, 507

Ontario, Productivity of Osprey, Pandion haliaetus, Nesting on Natural and Artificial Structures in the Kawartha Lakes, Ontario, 1991-2001, 58

Ontario River, Environment and Distribution of Age 0 Fishes in River Canard, a Lowland, 16

Ontario Watershed, Possible Tool Use by Beavers, Castor canadensis, in a Northern, 441
Ontario Woodlands, Detectability of Non-passerines Using "Pishing" in Eastern, 377

Onychomys, 95

Oporornis agilis, 70 philadelphia, 70

Opsopoeodus emiliae, 21

Opuntia polyacantha, 220

Orchid, Bracted Green, 428

Orconectes propinquus, 17

Oreamnos americanus, 197,574

Oregon Cascade Range, Apparent Predation by Gray Jays, Perisoreus canadensis, on Long-toed Salamanders, Ambystoma macrodactylum, in the, 291

Oreonetides spp., 264

flavescens, 264

rectangulatus, 264

rotundus, 264

vaginatus, 264

Oreophantes recurvatus, 264

Oriole, Baltimore, 70

Orobanche fasciculata, 418

Orodrassus canadensis, 259 vastus, 259

Osmia sp., 246 lignaria, 246 texana, 246

Osprey, 58,379

Osprey, Pandion haliaetus, Nesting on Natural and Artificial Structures in the Kawartha Lakes, Ontario, 19912001, Productivity of, 58

Ostrya, 96

Ottawa Field-Naturalists' Club Awards for 2004, The, 614

Ottawa Field-Naturalists' Club 11 January 2005, Minutes of the $126^{\text {th }}$ Annual Business Meeting of The, 474

Otter, European, 459

Nearctic River, 459

River, 323

Otters (Lontra canadensis) Contribute to Fall Scent Marking?, Do Juvenile Nearctic River, 459

Ovenbird, 71

Ovibos moschatus, 168

Ovis canadensis, 574

Owl, Barred, 367,379

Boreal, 367,578

Burrowing, 490

Eastern Screech, 578

Great Gray, 69

Great Horned, 62,71,395,578

Long-eared, 395

Northern Saw-whet, 367,578

Short-eared, 70,400

Snowy, 211

Owls, Aegolius acadicus, Caching Behavior by Wintering Northern Saw-Whet, 578

Owls, Asio otus: A Review of North American Banding Longeared, 395

Oxyptila americana, 270

Oxytrope, Arctic, 432

Jordal's, 432

Murray's, 432

Oxytropis arctica var. arctica, 417

arctica var. murrayi, 417

arctobia, 277

campestris ssp. jordalii, 418

deflexa ssp. foliosa, 277 
maydelliana, 277

nigrescens ssp. nigrescens, 418

sordida ssp. murrayi, 432

Ozyptila bryantae, 270

distans, 270

gertschi, 270

sincera canadensis, 270

Pachygnatha brevis, 268

Pagophilus groenlandicus, 382

Paintbrush, Scarlet, 433

Small-flowered, 434

Unalaska, 434

Pandion haliaetus, 58,379

Pandion haliaetus, Nesting on Natural and Artificial Structures in the Kawartha Lakes, Ontario, 1991-2001, Productivity of Osprey, 58

Panicum capillare var. occidentale, 439 dichotomiflorum, 548 miliaceum, 438

Papaver radicatum, 277 radicatum $\mathrm{ssp}$. kluanensis, 418

Papilio sp., 249

Paquet, P.C., 192

Parachnowitsch, A.L. and E. Elle. Insect Visitation to Wildflowers in the Endangered Garry Oak, Quercus garryana, Ecosystem of British Columbia, 245

Paralochloa, 497

Pardosa albomaculata, 267

concinna, 267

furcifera, 267

fuscula, 267

groenlandica, 267

hyperborea, 267

lapidicina, 267

mackenziana, 267

moesta, 267

saltuaria, 267

xerampelina, 267

Parelaphostrongylus tenuis, 334

Parmelia, 78

saxatilis, 78

sulcata, 78

Parnassia fimbriata, 418

Parrya arctica, 277,506

Partridge, Grey, 122

Partridge-foot, 431

Partridgeberry, 412

Parula americana, 69

Parus atricapillus, 367

Passer domesticus, 71

Passerculus sandwichensis, 70,570

Pear, C.A., 291

Peavine, Grass, 248

Pedicularis arctica, 289

capitata, 277,506

langsdorfii ssp. arctica, 277,506

lapponica, 277

macrodonta, 277

parviflora var. macrodonta, 289

Pelecopsis mengei, 264

Pelegrina flavipes, 268

montana, 268

Perca flavescens, 21,295

Perch, White, 18,295
Yellow, 20,295

Percina caprodes, 21

maculata, 21

Percursaria percursa, 82

Percursaria percursa from Hypersaline Springs in the Middle of the North American Continent, Identification of a Marine Green Alga, 82

Perdix perdix, 122

Perisoreus canadensis, 70,101,291

Perisoreus canadensis: Adaptations to Boreal Winters, Weight-carrying Ability and Caching Behavior of Gray Jays, 101

Perisoreus canadensis, on Long-toed Salamanders, Ambystoma macrodactylum, in the Oregon Cascade Range, Apparent Predation by Gray Jays, 291

Peromyscus sp., 446,452,578,589,591 gossypinus, 446

leucopus, 446,452,578 maniculatus, 194,323,414,445,451

Peromyscus maniculatus, in the Boreal Forest, Long-distance Movement of a Dispersing Deer Mouse, 451

Peromyscus maniculatus, in Wire Mesh Live-Traps: A Cautionary Note, Mortality of Deer Mice, 445

Peterson, T.S., A. Uesugi, and J. Lichter. Tree Recruitment Limitation by Introduced Snowshoe Hares, Lepus americanus, on Kent Island, New Brunswick, 569

Petrel, Leach's Storm, 570

Petrochilidon pyrrhonota, 70

Pewee, Eastern Wood, 71

Phaeophyscia hispidula, 78 sciastra, 78

Phalacrocorax auritus, 69

Phalaris arundinacea, 418

Phenacomys intermedius, 414

Pheucticus ludovicianus, 71

Phidippus borealis, 268 princeps, 268 purpuratus, 268 whitmanii, 268

Philanthus albopilosus, 9 politus, 10

Phillips, F.R., 381

Philodromus alascensis, 268 imbecillus, 268 mysticus, 268 pernix, 268 placidus, 268 rufus, 268 rufus quartus, 268

Phippsia algida, 418

Phlox gracilis, 248

Phlox, Slender, 248

Phoca hispida, 357 vitulina, 355

Pholcus phalangioides, 268

Phoxinus eos, 221,360 neogaeus, 360

Phragmites australis, 17,277 communis var. berlandieri, 280

Phyciodes mylitta, 249

Phyllodoce $\times$ intermedia, 418

Physcia caesia, 78 dubia, 78 phaea, 78

Physconia muscigena, 78 
Physidae, 547

Physocarpus, 96 opulifolius, 96

Picea sp., 135,208,457 engelmanii $\times$ glauca, 186 glauca, $77,186,255,381,404,451,458,574$ mariana, 186,255,333,381,412,457,574 rubens, 570 sitchensis, 160,197

Pickavance, J.R. and C.D. Dondale. An Annotated Checklist of the Spiders of Newfoundland, 254

Picoides arcticus, 367 dorsalis, 367 pubescens, $71,369,379$ villosus, 71,369,379

Pieris rapae, 249

Pike, Northern, 21

Pimephales notatus, 21,361 promelas, 21,221,351,361

Pimephales promelas, in New Brunswick, The Fathead Minnow, 351

Pin gris, 457 rouge, 457

Pine, Black, 224

Eastern White, 386

Jack, 332

Japanese Black, 224

Japanese Red, 224

Lodgepole, 186,419,451,574

Mugo, 224

Pitch, 9

Ponderosa, 132

Red, 8,38,237,332

Scotch, 8,231

Scots, 231,237

Shore, 344

White, 9,38,238,332,369,378

Pine, Pinus mugo, in Ontario, Identification and Status of the Introduced Black Pine, Pinus nigra, and Mugo, 224

Pine, Pinus nigra, and Mugo Pine, Pinus mugo, in Ontario, Identification and Status of the Introduced Black, 224

Pine, Pinus sylvestris, Replacing Corema, Corema conradii, Heathland in the Annapolis Valley, Nova Scotia, Invasive Scots, 237

Pinicola enucleator, 121

Pintail, Northern, 69,548

Pinus spp., 548

banksiana, 231,332,457

cembroides var. edulis, 231

contorta, 186,344,451,574

contorta ssp. latifolia, 418

densiflora, 224

edulis, 231

montana, 229

mugo, 224

mugo ssp. mugo, 229

mugo ssp. rotundata, 229

mugo ssp. uncinata, 229

nigra, 224

nigra var. nigra, 226

ponderosa, 132

resinosa, 8,38,231,237,332,457

rigida, 9

strobus, 9,38,238,332,369,378,386 sylvestris, 8,231,237

thunbergii, 224

Pinus тияo, in Ontario, Identification and Status of the Introduced Black Pine, Pinus nigra, and Mugo Pine, 224

Pinus nigra, and Mugo Pine, Pinus mugo, in Ontario, Identification and Status of the Introduced Black Pine, 224

Pinus sylvestris, Replacing Corema, Corema conradii, Heathland in the Annapolis Valley, Nova Scotia, Invasive Scots Pine, 237

Pipit, Sprague's, 490

Pirata bryantae, 267

canadensis, 267

cantralli, 267

insularis, 267

minutus, 267

montanus, 267

piraticus, 267

Pityohyphantes costatus, 264

limitaneus, 264

subarcticus, 264

Placidium squamulosum, 78

Planorbidae, 547

Plantago eriopoda, 418 major, 394

Plantain, Saline, 434

Platanthera clavellata, 388

Platycrista cheleusis, 587

Plecoptera, 547

Plectrophanax nivalis, 120

Plenoculus davisi, 10

Plethodon cinereus, 129

Pletscher, D.H., 446

Pleurozium schreberi, 586

Plover, Mountain, 532

Plovers, Charadrius montanus, Differential Parental Care by Adult Mountain, 532

Poa alpigena, 280

alpina, 277

glauca, 277

pratensis, 245

pratensis ssp. alpigena, 277

Pocadicnemis americana, 264

Podagrostis thurberiana, 420

Podiceps auritus, 69

Podilymbus podiceps, 69

Poecile spp., 121

atricapillus, 71

cinctus, 120

hudsonica, 70,367

major, 120

montanus, 120

palustris, 120

Poeciloneta sp., 264

furcata, 264

Pogonia ophioglossoides, 388

Polemonium pulcherrimum, 418

Polites themistocles, 12

Polygonum spp., 547

achoreum, 418

convolvulus, 418

lapathifolium, 418,547

pennsylvanicum, 548

pensylvanicum ssp. oneillii, 428

persicaria, 394,418,548

viviparum, 277 
Polytrichum, 78 lonchitis, 418

Pomoxis annularis, 21 nigromaculatus, 21

Pondhawk, Eastern, 234

Pondweed, Blunt-leaved, 279

Clasping-leaved, 28

Closed-leaved, 278

Curlyleaf, 546

Fine-leaved, 278

Horned, 28

Sago, 28

White-stemmed, 420

Ponera pennsylvanica, 39

Poplar, 41

Balsam, 77

Populus sp., 41,367,401,457

balsamifera, 77

deltoides, 17,132

tremuloides, 65,135,175,186,220,381,442,451,574

Porcupine, 125,159,177,453

Porcupines, Erethizon dorsatum, in Second-Growth Conifer Forests, The Influence of Thermal Protection on Winter Den Selection by, 159

Porrhomma sp., 264

gertschi, 264

terrestre, 264

Portulaca oleracea, 438

Porzana carolina, 69

Posidonia, 114

Potamogeton sp., 16,546

crispus, 546

dichotomiflorum, 547

epihydrus, 394

filiformis, 277

foliosus, 277

foliosus var. macellus, 278

natans, 548

obtusifolius, 276

pectinatus, 28

perfoliatus, 28

praelongus, 418

pusillus, 547

Potentilla anserina, 560

biflora, 277

bipinnatifida, 418

nivea ssp. nivea, 277

norvegica, 394

palustris, 277

recta, 394

potentille ansérine, 560

Pouliot, D., 105

Pouliot, D. et J.-F. Desroches. Découverte de la Salamandre à quatre orteils, Hemidactylium scutatum, à Québec, Québec: limite nord-est de l'espèce sur la rive nord du fleuve Saint-Laurent, 129

Powell, T., 451

Prairie-dog, Black-tailed, 532

Price, M.H.H., C.T. Darimont, N.N. Winchester, and P.C. Paquet. Facts from Faeces: Prey Remains in Wolf, Canis lupus, Faeces Revise Occurrence Records for Mammals of British Columbia's Coastal Archipelago, 192

Primrose, Siberian, 433

Primula nutans, 418 sibirica, 433

Proctor, H.C., 586

Procyon lotor, 62,298,453

Procyon lotor, in Eastern Ontario, Movements of Two Rabid Raccoons, 453

Proietto, R.L., 139

Proulx, G. Body Weights of Adult and Juvenile Northern Pocket Gophers, Thomomys talpoides, in Central Alberta Alfalfa Fields, 551

Proulx, G. Long-tailed Weasel, Mustela frenata, Movements and Diggings in Alfalfa Fields Inhabited by Northern Pocket Gophers, Thomomys talpoides, 175

Proulx, G. and R.M. Kariz. Winter Habitat Use by Moose, Alces alces, in Central Interior British Columbia, 186

Pruche du Canada, 129

Pruitt, W.O., Jr., Why and How to Study a Snowcover, 118

Prunus spp., 344

Psathyrostachys juncea, 418

Pseudacris triseriata, 291

Pseudocolpodium, 498

Pseudotsuga menziesii, 344

Ptarmigan, Willow, 44

Pteridium aquilinum, 240

Pterostichus melanarius, 271

Puccinellia, 497

agrostidea, 498

angustata, 497

arctica, 497

beringensis, 498

byrrangensis, 498

colpodioides, 498

jenisseiensis, 498

phryganodes, 497

poacea, 498

vahliana, 498

vahliana $\mathrm{X}, 498$

wrighti var. colpodioides, 498

wrighti var. flava, 498

wrighti var. wrightii, 498

wrightii, 498

Puccinellia, Addition to the Flora of Canada? A Specimen from the Arctic Archipelago, Northwest Territories Links Two Allopatric Species of Alkali Grass, 497

Puma concolor, 580

Puma concolor, in the Yukon, First Confirmation of Cougar, 580

Pumpkinseed, 21

Pusa hispida, 382

Pusillia mandibulata, 263

Pussytoes, Showy, 434

Pyrola secunda, 277

Pyrola, One-flowered, 288

Pyrrhula pyrrhula, 120

Quebec, Consequences of Beaver, Castor canadensis, Flooding on a Small Shore Fen in Southwestern, 385

Québec, et impact sur l'ichtyofaune, Charactéristiques d'une population introduit du Grand brochet, Esox lucius, dans le lac Ramsay, Parc de la Gatineau, 359

Québec, Extension de l'aire de distribution de la Couleuvre à collier, Diadophis punctatus edwardsii, dans l'est $\mathrm{du}, 457$

Québec: limite nord-est de l'espèce sur la rive nord du fleuve Saint-Laurent, Découverte de la Salamandre à quatre orteils, Hemidactylium scutatum, à Québec, 129 
Québec, Premières mentions et répartition de la Salamandre sombre du Nord, Desmognathus fuscus, sur la rive nord du fleuve Saint-Laurent, au, 105

Québec, Québec: limite nord-est de l'espèce sur la rive nord du fleuve Saint-Laurent, Découverte de la Salamandre à quatre orteils, Hemidactylium scutatum, à, 129

Québec, The Effect of Human Activity on Ant Species (Hymenoptera: Formicidae) Richness at the Mont St. Hilaire Biosphere Reserve, 38

Quercus spp., 9,343 alba, 368

bicolor, 368

garryana, 245,344

macrocarpa, 348

rubra, 38,129,240,348,368,386

Quercus garryana, Ecosystem of British Columbia, Insect Visitation to Wildflowers in the Endangered Garry Oak, 245

Quillback, 18

Quinn, N.W.S. Reconstructing Changes in Abundance of White-tailed Deer, Odocoileus virginianus, Moose, Alces alces, and Beaver, Castor canadensis, in Algonquin Park, Ontario, 1860-2004, 330

Quiscalus quiscula, 70

Raccoon, 62,298,453

Raccoons, Procyon lotor, in Eastern Ontario, Movements of Two Rabid, 453

Rail, Virginia, 69

Yellow, 69

Rallus limicola, 69

Ramalina intermedia, 78

Ramazzottius sp., 586

Rana luteiventris, 291 palustris, 578 pipiens, 389

Rangifer tarandus, 43,119,168,201,355,381

tarandus caribou, 124,136,573

tarandus fennicus, 124

tarandus granti, 124

tarandus groenlandicus, 124

tarandus pearyi, 120

tarandus tarandus, 124

Ranunculus aquatilis var. eradicatus, 277

occidentalis, 248

repens, 438

sabinei, 277

xspitzbergensis, 277

Raspberry, 369

Common, 44

Red, 570

Wild Red, 287

Rattlesnake, 95

Rauschia triangularis, 324

Raven, Common, 70,291,444

Reading, K.L., 276

Reddoch, A.H., 385

Reddoch, J.M. and A.H. Reddoch. Consequences of Beaver, Castor canadensis, Flooding on a Small Shore Fen in Southwestern Quebec, 385

Redhorse sp., 21 Shorthead, 21

Redpoll, 120 Common, 120

Redstart, American, 71

Reed, Common, 17,280
Reedgrass, Bluejoint, 77

Reeves, H.M., Review by, 307

Regulus calendula, 70 satrapa, 579

Reimchen, T.E., 202

Rheum rhabarbarum, 438

Rhinichthys cataractae, 221

Rhizocarpon disporum, 78

Rhizoplaca chrysoleuca, 78

Rhododendron groenlandicum, 412 lapponicum, 210

Rhynchospora alba, 277

Rhytidium rugosum, 80

Ribes spp., 369

Robertus banksi, 269

borealis, 269

fuscus, 269

riparius, 269

Robin, American, 70

Rogers, L.L. Weight-carrying Ability and Caching Behavior of Gray Jays, Perisoreus canadensis: Adaptations to Boreal Winters, 101

Rohner, C., 573

Rosa spp., 176

blanda, 277

woodsii, 418

Rosatte, R., M. Allan, R. Warren, P. Neave, T. Babin, L. Buchanan, D. Donovan, K. Sobey, C. Davies, F. Muldoon, and A. Wandeler. Movements of Two Rabid Rose, 176 Raccoons, Procyon lotor, in Eastern Ontario, 453

Western, 431

Rosebay, Lapland, 210

Rosemary, Bog, 210

Roussel, B., 457

Roy, J.F., Review by, 605

Rubus spp., 239,369

arcticus ssp. acaulis, 277

chamaemorus, 210,412

idaeus, 44,277,570

strigosus, 287

vermontanus, 240

Rugathodes aurantius, 269

sexpunctatus, 269

Rumex acetosella, 240

crispus, 438

maritimus ssp. fueginus, 418

Ruppia, 114

cirrhosa, 115

maritima, 28,115

occidentalis, 115

Ruppia, Widgeon Grass, balls in Saskatchewan Lakes, Occurrence, Composition and Formation of 114

Rush, Bog, 282

Swaying, 422

Thread, 427

Toad, 427

Rye, 422

Canadian Wild, 279

Russian Wild, 422

Sagebrush, 220,532

Sagina caespitosa, 277

nivalis, 418

Sagittaria, 390

latifolia, 394,548 
Salad, Corn, 248

Salamander, Long-toed, 291

Northwestern, 292

Salamanders, Ambystoma macrodactylum, in the Oregon Cascade Range, Apparent Predation by Gray Jays, Perisoreus canadensis, on Long-toed, 291

Salamandre à deux lignes, 106 à quatre orteils, 129 cendrées , 129 pourpre, 106 sombre du Nord, 105

Salamandre à quatre orteils, Hemidactylium scutatum, à Québec, Québec: limite nord-est de l'espèce sur la rive nord du fleuve Saint-Laurent, Découverte de la, 129

Salamandre sombre du Nord, Desmognathus fuscus, sur la rive nord du fleuve Saint-Laurent, au Québec, Premières mentions et répartition de la, 105

Salix sp., 65,77,96,132,135,175,186,208,220,441,506,574,591 alaxensis, 277

arctica, 277

arctica $\times$ glauca, 276

arctophila, 210,277

bebbiana, 96

brachycarpa ssp. niphoclada, 428

fuscescens, 277

glauca ssp. callicarpaea, 276

glauca var. acutifolia, 277

lanata ssp. richardsonii, 283

myrtillifolia, 276

niphoclada, 418

planifolia, 277

reticulata, 277

richardsonii, 277

rotundifolia, 276

rotundifolia ssp. rotundifolia, 283

sphenophylla, 277

Salmon, 591

Chinook, 591

Chum, 202

Coho, 197

Pacific, 202

Pink, 202

Salmon Carcasses: Opportunistic Use of a Seasonally Superabundant Food Source, Beavers, Castor canadensis, Feeding on, 591

Salmon, Oncorhynchus spp., as a Major Nutrient Source for Large Aggregations of Gulls, Larus spp., Post-Reproductive Pacific, 202

Salmonid, 353

Salticus scenicus, 268

Salvelinus fontinalis, 351

Sanderson, E.W., 1

Sandpiper, Least, 379

Spotted, 69,379

Upland, 69

Sandwort, Bog, 429

Sanicle, Pacific, 248

Sanicula crassicaulis, 248

Sapin baumier, 129,457

Sapsucker, Yellow-bellied, 71,367,379

Sarcobatus vermiculatus, 220

Sarsparilla, Wild, 44

Saskatchewan Lakes, Occurrence, Composition and FormaSaskatoon, 78
Satilatlas gertschi, 264 marxii, 264

Savannah Sparrow, 70

Saving the Wild: RENEW 2005, 613

Saxifraga nelsoniana ssp. carlottae, 417

nelsoniana ssp. pacifica, 418

nelsoniana ssp. porsildiana, 277

nivalis, 277

oppositifolia, 506

punctata ssp. carlottae, 431

punctata ssp. pacifica, 431

punctata ssp. porsildiana, 286

rivularis, 277

rufopilosa, 418

tricuspidata, 78,506

Saxifrage, Alpine, 286

Leather-leaved, 430

Prickly, 78

Schmiegelow, F.K.A., 573

Schmutz, J.K., 114

Schoenoplectus acutus, 418

acutus var. acutus, 427

pungens, 439

tabernaemontani, 418

Sciastes dubius, 262

truncatus, 264

Scirites pectinatus, 264

Scironis tarsalis, 265

Scirpus sp., 16

acutus, 418,548

americanus, 439

caespitosus ssp. austriacus, 277

cyperinus, 137,394

fluviatilis, 548

hudsonianus, 418

rollandii, 277

rufus, 417

validus, $17,418,548$

Sciurus carolinensis, 343,456 vulgaris, 121,343

Sciurus carolinensis, in British Columbia, The Distribution and Habitat Selection of Introduced Eastern Grey Squirrels, 343

Scolochloa festucacea, 77

Scolopax minor, 69

Scoter, 181

Black, 182

Surf, 182

White-winged, 182

Scoter, Melanitta spp., Distributions in Central Alaska Beaufort Sea Lagoons, 1999-2002, Long-tailed Duck, Clangula hyemalis, Eider, Somateria spp., and, 181

Scotinotylus pallidus, 265 sacer, 265

Sculpin, 582

Scutellaria galericulata, 394

Scyletria inflata, 265

Seal, Grey, 355

Harbour, 355

Harp, 382

Hooded, 382

Ringed, 357,382

Seburn, D., Review by, 145

Secale cereale, 418

Sedge, 16,186,211,591 
Awned, 77

Bebb's, 423

Blackish, 426

Bristle-leaved, 424

Bristly, 137

Brownish, 423

Buxbaum's, 423

Dunhead, 426

Enander's, 425

Few-seeded Fen, 425

Grey, 424

Hay, 426

Kellogg's, 425

Pale, 425

Parry's, 426

Sartwell's, 426

Slender, 424

Small-winged, 425

Spikehead, 426

Weak, 425

Seiurus aurocapillus, 71 noveboracensis, 69

Semljicola obtusus, 265

Semotilus atromaculatus, 295,351,361

Senecio congestus, 277

Serfass, T.L., 459

Sericocarpus rigidus, 245

Setaria spp., 548 glauca, 438

Setophaga ruticilla, 71

Shad, Gizzard, 18

Shark, Bluntnose Sixgill, 537 Greenland, 355

Sharks, Hexanchus griseus, at Flora Islets, British Columbia, Body Size Distribution and Frequency of Anthropogenic Injuries of Bluntnose Sixgill, 537

Sharks, Somniosus microcephalus, Vision and its Relationship to Novel Behaviour in St. Lawrence River Greenland, 355

Sheep, 200

Bighorn, 574

Shepherdia azgenteas, 220 canadensis, 276

Shik, J.Z., A. Francoeur, and C.M. Buddle. The Effect of Human Activity on Ant Species (Hymenoptera: Formicidae) Richness at the Mont St. Hilaire Biosphere Reserve, Québec, 38

Shiner, Common, 21

Emerald, 20

Golden, 21,295

Mimic, 21

Pugnose, 18

Sand, 21

Spotfin, 18

Spottail, 21

Shootingstar, Broad-leaved, 248

Shoveler, Northern, 69,548

Shrew, 122,578

Masked, 43,324,579

Pygmy, 414

Short-tailed, 456,578

Shrew, Blarina brevicauda, Apparently Killed by Red Squirrel, Tamiasciurus hudsonicus, Short-tailed, 456

Shrike, 102,578

Loggerhead, 490
Silene acaulis ssp. subacaulescens, 418

cserei, 439

walensis, 277

Silverside, Brook, 21

Singa variabilis, 257

Sisicottus montanus, 265 quoylei, 265

Sisicus apertus, 265 penifusifer, 265

Sisis rotundus, 265

Siskin, Pine, 71

Sitta canadensis, 71,367 carolinensis, 367

Sitticus cutleri, 268

finschii, 268

floricola palustris, 268

palustris, 268

ranieri, 268

striatus, 268

Skunk, 454 Striped, 584

Slough, B.G., 589

Smilacina trifolia, 44

Smith, D.G., 578

Snake, Garter, 292

Snap-dragon, Dwarf, 438

Snipe, Wilson's, 69

Snowberry, Creeping, 44

Soapberry, 287

Sobey, K., 453

Solenopsis molesta, 39

Solidago sp., 388

altissima, 525

bicolor, 240

canadensis, 390

graminifolia var. major, 277

juncea, 240

uliginosa, 388

Solomon's Seal, Three-Leaved False, 44

Somateria spp., 181

mollissima v-nigrum, 182

spectabilis, 182

Somateria spp., and Scoter, Melanitta spp., Distributions in Central Alaska Beaufort Sea Lagoons, 1999-2002, Long-tailed Duck, Clangula hyemalis, Eider, 181

Somniosus microcephalus, 355

Somniosus microcephalus, Vision and its Relationship to Novel Behaviour in St. Lawrence River Greenland Sharks, 355

Sonchus arvensis ssp. uliginosus, 439 arvensis var. glabrescens, 439

Sora, 69 uliginosus, 438

Sorbus americanus, 570 sitchensis, 418

Sorex sp., 122,578 cinereus, $43,324,579$ hoyi, 414

Souessa spinifera, 265

Sougambus bostoniensis, 265

Soulgas corticarius, 265

Soybean, 547

Sparganium sp., 16,390

americanum, 137,389

hyperboreum, 277 
Sparrow, 578

Chipping, 71

House, 71

LeConte's, 69

Lincoln's, 70

Savannah, 570

Song, 70,120,578

Swamp, 69

White-crowned, 71

White-throated, 70

Spartina gracilis, 277

Spermophilus richardsonii, 442

Sphagnum sp., 129,388,404

magellanicum, 388

teres, 388

Sphaigne, 129

Sphixapata vigilans, 13

Sphyrapicus varius, 71,367,379

Spider, 95,254

Spiders of Newfoundland, An Annotated Checklist of the, 254

Spike-rush, Bald, 439

Spiraea betulifolia, 438

Spirembolus oreinoides, 265

Spizella passerina, 71

Spreadwing, Northern, 234

Spruce, 135,186,208

Black, 186,255,333,381,412,574

Red, 570

Sitka, 160,197

White, 77,186,255,381,404,451,570,574

Squirrel, Douglas, 344

Eastern Grey, 343

European Red, 343

Grey, 456

North American Red, 121,343

Northern Flying, 344,591

Red, 43,121,323,456,591

Richardson's Ground, 442

Southern Flying, 374

Squirrel, Tamiasciurus hudsonicus, Short-tailed Shrew, Blarina brevicauda, Apparently Killed by Red, 456

Squirrels, Sciurus carolinensis, in British Columbia, The Distribution and Habitat Selection of Introduced Eastern Grey, 343

St. John's-wort, 14

Starling, European, 71

Starwort, Umbellate, 429

Staurothele, 76

drummondii, 78

fissa, 78

Steatoda bipunctata, 269

Stellaria calycantha, 277

longipes, 277

umbellata, 418

Stemonyphantes blauveltae, 265

Stenamma diecki, 39 impar, 39

Steniolia, 6

Stercorarius parasiticus, 211

Stevens, S.D. and T.I. Wellicome. A Survey for Federally Listed Grassland Birds at First Nations Reserves, 490

Stevens, S.S., 459

Stickleback, Brook, 221

Threespine, 28
Stictiella callista, 13

corniculata, 13

emarginata, 6

evansi, 13

formosa, 13

pulchella, 13

pulchella pulchella, 13

pulchella serrata, 13

serrata, 13

Stictiella emarginata (Hymenoptera: Sphecidae)*, Nesting Behavior, Ecology, Seasonal and Geographic Distribution of the Sand Wasp, 6

Stipa comata, 220

Stocek, R.F., 351

Stoneroller, Central, 18

Stonewort, 16

Strix nebulosa, 69 varia, 367,379

Stuckenia spp., 546 filiformis, 548 pectinata, 28,546

Sturnella magna, 71

Sturnus vulgaris, 71

Styloctetor stativus, 265

Sucker, White, 18,221,295

Sundew, Great, 430

Sunfish, Green, 21

Longear, 18 Orangespotted, 18

Swallow, Barn, 70

Cliff, 70

Tree, 70

Swallow-wort, Pale, 525

Swallow-wort, Vincetoxicum rossicum, following Aboveground Tissue Loss: Implications for the Timing of Mechanical Control, Response of Pale, 525

Swertia perennis, 417

Swertia, Alpine Bog, 433

Sylvester, R.M., S.E. Freeling, and C.R. Berry, Jr. First Record of the Plains Minnow, Hybognathus placitus, in Canada, 219

Symphoricarpos accidentalis, 220

Symphyotrichum ciliatum, 418

lanceolatum ssp. hesperium var. hesperium, 439 laurentianum, 556 yukonense, 418

Symphyotrichum laurentianum, aux Îles-de-la-Madeleine, Protocole de suivi des populations d'aster du SaintLaurent, 556

Symplocarpus foetidus, 129

Syrphus, 250

Tachycineta bicolor, 70

Tachysphex pechumani, 10 similis, 10 tarsatus, 10

Taenia pisiformis, 324

Tamarack, 255,404,574

Tamias striatus, 323,578

Tamiasciurus douglasii, 344 hudsonicus, 43,121,323,343,456,591

Tamiasciurus hudsonicus, Short-tailed Shrew, Blarina brevicauda, Apparently Killed by Red Squirrel, 456

Tapinocyba bicarinata, 265 exigua, 264 
flagellata, 265

lindrothi, 265

minuta, 265

scopuliferum, 262

simplex, 265

Tapinoma sessile, 39

Tapinopa bilineata, 265

Taraxacum spp., 552 lyratum, 418

Tardigrada, 586

Taxidea taxus, 442,584

Taxus canadensis, 457

Teal, Blue-winged, 69,379,547 Green-winged, 69,547

Tegenaria atrica, 256 domestica, 256 duellica, 256

Tennesseellum formicum, 265

Tern, Black, 69,78

Tête-de-boule, 361

Tetragnatha caudata, 255 elongata, 268

extensa, 269

laboriosa, 269

vermiformis, 255 versicolor, 269

Tetramorium caespitum, 39

Tetrao parviventris, 120 tetrix, 120

Texas, Swift Fox, Vulpes velox, Den Located Next to a Railroad Track in Northwestern, 584

Thamnophis sp., 292 sirtalis, 458

Thanatus formicinus, 268

Theberge, J.B., Review by, 308

Thellungiella salsuginea, 429

Thelypteris palustris, 388

Theonoe stridula, 269

Theridion aurantium, 269 differens, 269 glaucescens, 269 montanum, 269 murarium, 269 petraeum, 270 pictum, 270 sexpunctatum, 269 tectum, 269

Theridiosoma gemmosum, 255 radiosum, 270

Theridula emertoni, 270 sphaerula, 270

Thistle, Elk, 435 Leafy, 435

Thomomys spp., 175 bottae, 551 talpoides, 175,551

Thomomys talpoides, in Central Alberta Alfalfa Fields, Body Weights of Adult and Juvenile Northern Pocket Gophers, 551

Thomomys talpoides, Long-tailed Weasel, Mustela frenata, Movements and Diggings in Alfalfa Fields Inhabited by Northern Pocket Gophers, 175

Thomson, E.R. Papillate Watermeal, Wolffia brasiliensis, in Eastern Ontario: An Addition to the Flora of Canada, 137
Thomson, J.A., 214

Thorn, T.D., R.B. Emery, D.W. Howerter, J.H. Devries, and B.L. Joynt. Use of Radio-Telemetry to Test for Investigator Effects on Nesting Mallards, Anas platyrhynchos, 541

Thrasher, Brown, 71

Thrush, Hermit, 70

Swainson's, 70

Wood, 70

Thuidium abietinum, 80

Thuja occidentalis, 386,404 plicata, 160,344

Thymoites oleatus, 270

Thyreosthenius parasiticus, 265

Tibellus maritimus, 268

Tick, Winter, 330

Tigellinus tricornis, 266

Tilia, 96

americana, 369

americana var. americana, 96

Timmins, C.A., 16

Timoney, K.P. and J. Marsh. Lichen Trimlines in the PeaceAthabasca Delta: Variations in Flora, Form, and Disturbance Regime, 76

Tiso vagans, 265

Tit, Great, 120

Greyheaded, 120

Long-tailed, 120

Marsh, 120

Willow, 120

Titmouse, Tufted, 374

Toad, Western, 291

Tofieldea coccinea, 277 glutinosa $\mathrm{ssp}$. brevistyla, 428 pusilla, 277

Tokaryk, T., Reviews by, 305,306

Tolypella glomerata, 26

Torreyochloa pallida var. fernaldii, 394

Tortula ruralis, 80

Toxostoma rufum, 71

Trachynella nudipalpis, 266

Tramea lacerata, 234

Tree, Cherry, 344

Trematode, 324

Triadenum virginicum, 388

Triantha glutinosa, 418

Trichophorum alpinum, 418

Trichopterna mengei, 264

Trichostrongylus axei, 324

Trientalis borealis, 240

Triglochin maritimum, 278

Tringa flavipes, 69,379 melanoleuca, 379

Trisetum spicatum, 278

Triteleia grandiflora var. howellii, 248

Trochosa terricola, 267 terricola pratensis, 267

Troglodytes aedon, 374 troglodytes, 69

Tropaeolum sp., 438

Trout, Brook, 351

Tscherter, U., 214

Tsuga canadensis, 38,129,332,386 heterophylla, 160,197,344 mertensiana, 291 
Tunagyna debilis, 265

Turdus migratorius, 70

Tursiops sp., 217

Turtle, Blanding's, 389

Painted, 389

Twinberry, Black, 434

Twinflower, 289

Tympanuchus cupido, 507,515

cupido cupido, 516

cupido pinnatus, 523

cupido $\times$ phasianellus, 507,515

phasianellus, 507

phasianellus campestris, 507,515

phasianellus phasianellus, 507,515

Tympanuchus cupido $\times$ phasianellus, Hybridization on Manitoulin Island, Ontario, "Prairie Grouse", 507

Tympanuchus cupido $\times$ phasianellus, of Manitoulin Island, Ontario, Plumage and Internal Morphology of the "Prairie Grouse", 515

Typha spp., 221,403

angustifolia, 16

latifolia, 16,72,389,418

Typhocrestus pygmaeus, 265

Uesugi, A., 569

Ulmus, 96

americana, 96,370

rubra, 370

Ulothrix, 83

Ulva intestinalis, 83

Umbilicaria, 78 deusta, 78 muehlenbergii, 78

Umbra limi, 21,361

Umbre de vase, 361

Ursus americanus, 50,164,298,323,339,381,574 arctos, $339,449,574$

Ursus americanus, Ecology on the Northeast Coast of Labrador, Black Bear, 164

Ursus arctos, from Coastal British Columbia, Observations of Autumn Courtship and Breeding in Brown Bears, 449

Usui, M., P.G. Kevan, and M. Obbard. Pollination and Breeding System of Lowbush Blueberries, Vaccinium angustifolium Ait. and V. myrtilloides Michx. (Ericacaeae), in the Boreal Forest, 48

Utricularia cornuta, 388

macrorhiza, 137

minor, 418

Vaccinium spp., 44,48

angustifolium, 48,239

myrtilloides, 48

uliginosum, 412

vitis-idaea, 210,412

vitis-idaea var. minus, 278

Vaccinium angustifolium Ait. and V. myrtilloides Michx. (Ericacaeae), in the Boreal Forest, Pollination and Breeding System of Lowbush Blueberries, 48

Vaccinium myrtilloides Michx. (Ericacaeae), in the Boreal Forest, Pollination and Breeding System of Lowbush Blueberries, Vaccinium angustifolium Ait. and, 48

Vachon, J., B.F. Lavallée, et F. Chapleau. Charactéristiques d'une population introduit du Grand brochet, Esox lucius, dans le lac Ramsay, Parc de la Gatineau, Québec, et impact sur l'ichtyofaune, 359
Valerian, Sitka, 434

Valeriana sitchensis, 418

Valerianella locusta, 248

Vallisneria americana, 16,546

Veery, 70

Ventre citron, 360 pourri, 361 rouge du nord, 360

Verbena hastata, 418

Vermivora peregrina, 71 ruficapilla, 70

Vermontia thoracica, 265

Veronica americana, 418

Verrucaria, 76

Vetch, American, 432 Common, 248

Viburnum alnifolium, 129 cassinoides, 129 nudum var. cassinoides, 240

Vicia americana, 418 sativa, 248

Vincetoxicum rossicum, 525

Vincetoxicum rossicum, following Aboveground Tissue Loss: Implications for the Timing of Mechanical Control, Response of Pale Swallow-wort, 525

Vine, Dog-strangling, 525

Viola adunca, 418 macloskeyi ssp. pallens, 389 praemorsa ssp. praemorsa, 248

Violet, Hook-spur, 432 Yellow Montane, 248

Viorne à feuilles d'aulne, 129 cassinoïde, 129

Vireo gilvus, 70 olivaceus, 71 philadelphicus, 71 solitarius, 71

Vireo, Blue-headed, 71 Philadelphia, 71

Red-eyed, 71 Warbling, 70

Vole, 122,395

Heather, 414

Long-tailed, 445

Meadow, 43,323,414,446

Northern Red-backed, 445

Red-backed, 323

Rock, 412

Southern Red-backed, 43,414 Taiga, 446

Vole, Microtus chrotorrhinus, in Labrador, A Range Extension for the Rock, 412

Volucella bombylans, 249

Vulpes macrotis mutica, 134 velox, 134,584 vulpes, 120,323

Vulpes macrotis mutica, Aggressive Behaviour Exhibited by a San Joaquin Kit Fox, 134

Vulpes velox, Den Located Next to a Railroad Track in Northwestern Texas, Swift Fox, 584

Wabasso cacuminatus, 265 quaestio, 265

Walckenaeria arctica, 266 atrotibialis, 266 
auranticeps, 266

castanea, 266

clavipalpis, 266

communis, 266

cuspidata, 266

digitata, 266

directa, 266

exigua, 266

karpinskii, 266

lepida, 266

pallida, 266

palustris, 266

redneri, 266

spiralis, 266

tricornis, 266

Walmus borealis, 256

Walnut, Black, 348

Wandeler, A., 453

Warbler, Black-and-white, 70

Black-throated Blue, 70

Black-throated Green, 70

Cape May, 71

Chestnut-sided, 71

Connecticut, 70

Magnolia, 70

Mourning, 70

Nashville, 70

Northern Parula, 69

Palm, 69

Tennessee, 71

Wilson's, 69

Yellow, 70

Yellow-rumped, 70

Warner, B.G., 64

Warren, R., 453

Wasp, Sand, 6

Wasp, Stictiella emarginata (Hymenoptera: Sphecidae)*, Nesting Behavior, Ecology, Seasonal and Geographic Distribution of the Sand, 6

Water-bear, 586

Water-bears from the Rocky Mountains: A First Look at Alberta's Tardigrade Fauna, 586

Water-horehound, Tuberous, 288

Watermeal, 137

Watermeal, Wolffia brasiliensis, in Eastern Ontario: An Addition to the Flora of Canada, Papillate, 137

Water-milfoil, Verticillate, 432

Watershield, 137

Waterthrush, Northern, 69

Waxwing, Cedar, 70

Way, J.G. and R.L. Proietto. Record Size Female Coyote, Canis latrans, 139

Weasel, Long-tailed, 175

Short-tailed, 175,323

Weasel, Mustela frenata, Movements and Diggings in Alfalfa Fields Inhabited by Northern Pocket Gophers, Thomomys talpoides, Long-tailed, 175

Weir, R.D., 296

Wellicome, T.I., 490

Wendland, J.M., 591

Wersal, R.M., B.R. McMillan, and J.D. Madsen. Food Habits of Dabbling Ducks During Fall Migration in a Prairie Pothole System, Heron Lake, Minnesota, 546

Whale, Beluga, 214

Blue, 214

Fin, 214
Humpback, 214

Minke, 214

Whales, Balaenoptera acutorostrata, in the SaguenaySt. Lawrence National Marine Park, Novel Surface Feeding Tactics of Minke, 214

Wheatgrass, Slender, 78

Whitefaces, 234

Whitefish, Atlantic, 294

Whitefish, Coregonus huntsmani, from the Wild, First Record of Age 0+ Atlantic, 294

Whitlow-grass, Alaska, 429

Few-seeded, 430

Gray-leaved, 430

Rocky Mountain, 430

Starflowered, 430

Wigeon, American, 69

Widgeon-grass, 28

Widgeon Grass, balls in Saskatchewan Lakes, Occurrence, Composition and Formation of Ruppia, 114

Willow, 65,77,132,135,175,186,208,220,441,574,591

Arctic, 282

Barren-ground, 428

Bebb's, 96

Blue-green, 282

Blueberry, 283

Net-veined, 283

Richardson's, 283

Round-leaf, 283

Tea-leaf, 283

Willowherb, Hornemann's, 432

Swamp, 288

Wilsonia pusilla, 69

Winchester, N.N., 192

Wolf, 135,139,192,197,330,381,446,573

Eastern Canadian, 339

Gray, 1,101,197,442

Newfoundland, 323

Timber, 101

Wolf, Canus lupus, Den and Associated Human Activity in the Southwestern Yukon Territory, An Ancient, 135

Wolf, Canis lupus, Faeces Revise Occurrence Records for Mammals of British Columbia's Coastal Archipelago, Facts from Faeces: Prey Remains in, 192

Wolf, Canis lupus, near Icy Bay, Alaska, Attempted Predation of a Child by a Gray, 197

Wolffia arhiza, 137

borealis, 137

brasiliensis, 137

columbiana, 137

papulifera, 137

punctata, 137

Wolffia brasiliensis, in Eastern Ontario: An Addition to the Flora of Canada, Papillate Watermeal, 137

Wolverine, 120

Wolves, Canis lupus, in Relation to Ungulate Kill Sites in Westcentral Alberta, Travel Rates of, 573

Wolves, Canis lupus, in the Wild, Longevity and Productivity of Three, 446

Wood-rush, Piper's, 427 Spiked, 427

Woodcock, American, 69

Woodland Star, Small-flowered, 248

Woodley, E., Review by, 309

Woodpecker, American Three-toed, 367

Black-backed, 367

Downy, 71,369,379 
Hairy, 71,369,379

Pileated, 70,367,379

Red-bellied, 369

Red-headed, 369

Woodpecker Nest Tree Characteristics in Upper Midwestern Oak Forests, 367

Woodsia ilvensis, 278

Woodsia, Rusty, 278

Wool-grass, 137

Worm, Meningeal, 334

Wormwood, Dragon, 438

Wren, House, 374

Marsh, 69

Winter, 69

Wubana drassoides, 266 pacifica, 266

Xanthoparmelia somloënsis, 78

Xanthoria elegans, 78

$\times$ Ceratinops annulipes, 261

Xerostictia, 6

Xysticus canadensis, 270

discursans, 270

elegans, 270

emertoni, 270

keyserlingi, 270

labradorensis, 270

luctuosus, 270

obscurus, 270

triguttatus, 270

Yarrow, 248,438

Pearl, 438

Yellowhammer, 120

\section{Index to Book Reviews}

\section{Botany}

Blouin, G. An Eclectic Guide to Trees East of the Rockies, 497

Douglas, G.W., G.B. Straley, D. Meidinger and J. Pojar. Illustrated Flora of British Columbia Volumes 1-8, 329

Evans, C.L. The War on Weeds in the Prairie West: An Environmental History, 670

Kershaw, L. Ontario Wild Flowers, 330

Kirk, P.M., P.F. Cannon, J.C. David and J.A. Stalpers. Ainsworth and Bisby's Dictionary of the Fungi, 328

Lesica, P. A Flora of Glacier National Park, Montana, 159

Mitchell, R.S. and L. Danaher. Northeastern Fern Identifier, 669

More, D. and J. White. The Illustrated Encyclopedia of Trees, 496

Riley, J.L. Flora of the Hudson Bay Lowland and its Postglacial Origins, 669

Schnell, D.E. Carnivorous Plants of the United States and Canada: Second Edition, 496

\section{Environment}

Bierregaard, R.O. Jr., C. Gascon, T.E. Lovejoy, and R. Mesquita. Lessons from Amazonia: The Ecology and Conservation of a Fragmented Forest, 672

DeGraaf, R.M. Trees, Shrubs, and Vines for Attracting Birds, 160
Yellowlegs, Greater, 379

Lesser, 69,379

Yellowthroat, Common, 69

Yucca sp., 584

Yucca, 584

Yukon, First Confirmation of Cougar, Puma concolor, in the, 580

Yukon Territory, An Ancient Wolf, Canus lupus, Den and Associated Human Activity in the Southwestern, 135

Yukon Territory VII, New Records of Vascular Plants in the, 417

Zannichellia spp., 548 palustris, 28

Zapus sp., 578

Zea mays, 548

Zelotes fratris, 259 subterraneus, 259 sula, 259

Zenaida macroura, 71,379

Zielinski, R., 537

Zimmerling, J.R. Detectability of Non-passerines Using "Pishing" in Eastern Ontario Woodlands, 377

Zimmerling, T.N. The Influence of Thermal Protection on Winter Den Selection by Porcupines, Erethizon dorsatum, in Second-Growth Conifer Forests, 159

Zonotrichia leucophrys, 71

Zonotrichia albicollis, 70

Zornella cultrigera, 266

Zostera marina, 28

Zygadenus venenosus, 248

Zygiella atrica, 257

montana, 257

nearctica, 257

Dixon, T.F. City Wilds: Essays and Stories about Urban Nature, 498

Donovan, T.M. and C.W. Welden. Spreadsheet Exercises in Conservation Biology and Landscape Ecology, 506

Donovan, T.M. and C.W. Welden. Spreadsheet Exercises in Ecology and Evolution, 506

D'Orso, M. Plundering Paradise, 335

Ducey, J.E. Birds of the Untamed West: The History of Birdlife in Nebraska, 1750 to 1875,336

Franklin, S.E. Remote Sensing for Sustainable Forest Management, 330

Frelich, L.E. Forest Dynamics and Disturbance Regimes: Studies from Temperate Evergreen-Deciduous Forests, 502

Higgs, E. Nature by Design, 505

Johnsgard, P.A. Great Wildlife of the Great Plains, 504

Jones, H.G., J.W. Pomeroy, D.A. Walker and R.W. Hoham. Snow Ecology: An Interdisciplinary Examination of Snow-Covered Ecosystems, 332

Larson, D., U. Matthes, and P. Kelly. Cliff Ecology: Pattern and Process in Cliff Ecosystems, 672

Morris, W.F. and D.F. Doak. Quantitative Conservation Biology: Theory and Practice of Population Viability Analysis, 507

Nadkarni, N.M. and N.T. Wheelwright. Monteverde: Ecology and Conservation of a Tropical Cloud Forest, 334 
Pullin, A.S. Conservation Biology, 499

Schwartzman, D. Life, Temperature, and the Earth, 498

Song, S.J. Ecological Basis for Stand Management: A Summary and Synthesis of Ecological Responses to Wildfire and Harvesting in Boreal Forests, 500

Suzuki, D. and A. McConnell. The Sacred Balance: A Visual Celebration of Our Place in Nature, 503

Thomashow, M. Bringing the Biosphere Home, Learning to Perceive Global Environmental Change, 159

Watt, A. The Last Island: A Naturalist's Sojourn on Triangle Island, 505

Wiese, F. Seabirds and Atlantic Canada's Ship-Source Oil Pollution, 332

\section{Miscellaneous}

Arnaudin, M.P. A Bird in the Bush: The Story of the Province of Quebec Society for the Protection of Birds 19172002, 508

Baker, D.B. John Keast Lord: Materials for a Life, 338

Berry, M.F. The Dinosaur Filmography, 341

Browne, J. Charles Darwin, The Power of Place, 340

Debus, A.A. and D.E. Debus. Paleoimagery, The Evolution of Dinosaurs in Art, 341

Hodgson, B. Naturalists: A Journal, 342

Hodgson, B. Women Travelers: A Journal, 342

King, J. Farley: The Life of Farley Mowat, 509

Lawrence, R.D. The North Runner, 674

Nicklen, P. and H. Brody. Seasons of the Arctic, 674

Stroud, P.T. The Emperor of Nature: Charles-Lucien Bonaparte and his World, 337

Suzuki, D. When the Wild Comes Leaping Up: Personal Encounters with Nature, 339,675

\section{Zoology}

Barter, M.A., Shorebirds of the Yellow Sea: Importance, Threats and Conservation Status, 321

Behnke, R.J. Trout and Salmon of North America, 318

Brooks, R.J., D. Strickland, and R.J. Rutter. Reptiles and Amphibians of Algonquin Provincial Park, 490

Byers, J.A. Built for Speed: A Year in the Life of Pronghorn, 661

Catling, P.M., C.D. Jones and P. Pratt. Ontario Odonata. Volume 3 (including observations for the year 2001), 487

Clements, J.F. Birds of the World: A Checklist, 322

Collette, B.B. and G. Klein-MacPhee. Bigelow and Schroeder's Fishes of the Gulf of Maine, 319

del Hoyo, J., A. Elliott, and J. Sargatal. Handbook of Birds of the World. Volume 8: Broadbills to Tapaculos, 663

Downer, J. Weird Nature: An Astonishing Exploratioin of Nature's Strangest Behavior, 158

Eder, T. Mammals of Ontario, 487

Ernst, C.H. and E.M. Ernst. Snakes of the United States and Canada, 495

Fishpool, L.D.C. and M.I. Evans. Important Bird Areas in Africa and Associated Islands: Priority Sites for Conservation, 664
Gittleman, J.L., S.M. Funk, D. MacDonald, and R.K. Wayne. Carnivore Conservation, 155

Halliday, T. and K. Adler. Firefly Encyclopedia of Reptiles and Amphibians, 327

Heintzelman, D. All-Weather Hawk Watcher's Field Journal, 326

Holman, J.A. In Quest of Great Lakes Ice Age Vertebrates, 494

Houston, C.S. and W. Anaka. Birds of Yorkton - Duck Mountain, 668

Jaramillo, A. Birds of Chile, 659

Jones, K.A. Knowing Bass: The Scientific Approach to Catching More Fish, 156

Kays, R.W. and D.E. Wilson. Mammals of North America, 488

Knott, D.L. A Spring Expedition to the Falkland Islands and Antarctica, 320

Lacey, E.A., J.L. Patton, and G.N. Cameron. Life Underground, the Biology of Subterranean Rodents, 157

Leverton R. Enjoying Moths, 327

Lieske, E. and R. Myers. Coral Reef Fishes: Indo-Pacific and Caribbean - Revised Edition, 662

Love, M.S., M. Yoklavich and L. Thorsteinson. The Rockfishes of the Northeast Pacific, 323

Manly, B.J., L.L. McDonald, D.L. Thomas, T.L. McDonald and W.P. Erickson. Resource Selection by Animals, 325

Matthiessen, P. The Birds of Heaven: Travels with Cranes, 158

Maxwell, B.A., J.K. Werner, P. Hendricks, and D.L. Faith. Herpetology in Montana: A History, Status Summary, Checklists, Dichotomous Keys, Accounts of Native, Potentially Native, and Exotic Species, and Indexed Bibliography, 491

Mecklenburg, C.W., T.A. Mecklenburg, and L.K. Thorsteinson. Fishes of Alaska, 151

Riede, K. Global Register of Migratory Species (GROMS): Database, GIS Maps, and Threat Analysis, 154

Rossi, J. and R. Rossi. Snakes of the United States and Canada: Natural History and Care in Captivity, 666

Russell, C. and M. Enns. Grizzly Heart: Living without Fear among the Brown Bears of Kamchatka, 662

Saffron, I. Caviar: The Strange History and Uncertain Future of the World's Most Coveted Delicacy, 324

Scott, J.M., S. Conant and C. Van Riper III. Evolution, Ecology, Conservation, and Management of Hawaiian Birds: A Vanishing Avifauna, 153

Shirihai, H. The Complete Guide to Antarctic Wildlife, 489

Sibley, D.A. Sibley's Birding Basics, 151

Szczerbak, N.N. Guide to the Reptiles of the Eastern Palearctic, 492

Taylor, P. The Birds of Manitoba, 660

Urquhart, E. and A. Bowley. Stonechats: A Guide to the Genus Saxicola, 667

Zug, G.R., L.J. Vitt, and J.P. Caldwell. Herpetology: An Introductory Biology of Amphibians and Reptiles, Second Edition, 493 